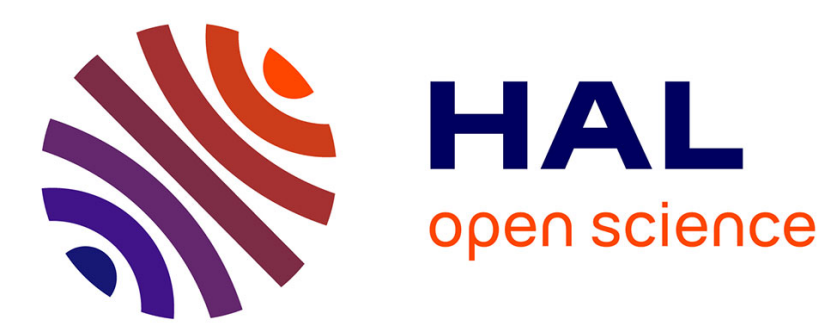

\title{
Nonintrusive reduced-order modeling of parametrized time-dependent partial differential equations
}

Christophe Audouze, Florian de Vuyst, Prasanth Nair

\section{To cite this version:}

Christophe Audouze, Florian de Vuyst, Prasanth Nair. Nonintrusive reduced-order modeling of parametrized time-dependent partial differential equations. 2010. hal-00530847v2

\section{HAL Id: hal-00530847 \\ https://hal.science/hal-00530847v2}

Preprint submitted on 14 Nov 2010

HAL is a multi-disciplinary open access archive for the deposit and dissemination of scientific research documents, whether they are published or not. The documents may come from teaching and research institutions in France or abroad, or from public or private research centers.
L'archive ouverte pluridisciplinaire HAL, est destinée au dépôt et à la diffusion de documents scientifiques de niveau recherche, publiés ou non, émanant des établissements d'enseignement et de recherche français ou étrangers, des laboratoires publics ou privés. 


\title{
Nonintrusive reduced-order modeling of parametrized time-dependent partial differential equations
}

\author{
Christophe Audouze ${ }^{\dagger}$, Florian De Vuyst ${ }^{\dagger}$, Prasanth B. Nair ${ }^{\dagger *}$ \\ $\dagger$ Computational Engineering and Design Group, School of Engineering Sciences \\ University of Southampton, Highfield, Southampton SO 17 1BJ, United Kingdom \\ Laboratoire CMLA, ENS Cachan \\ 61 avenue du Président Wilson 92235 Cachan, France
}

\begin{abstract}
We propose a non-intrusive reduced-order modeling method based on the notion of space-timeparameter proper orthogonal decomposition for approximating the solution of non-linear parametrized time-dependent partial differential equations. A two-level proper orthogonal decomposition method is introduced for constructing spatial and temporal basis functions with special properties such that the reduced-order model satisfies the boundary and initial conditions by construction. A radial basis function approximation method is used to estimate the undetermined coefficients in the reduced-order model without resorting to Galerkin projection. This nonintrusive approach enables the application of our approach to general problems with complicated nonlinearity terms. Numerical studies are presented for the parametrized Burgers' equation and a parametrized convection-reaction-diffusion problem. We demonstrate that our approach leads to reduced-order models that accurately capture the behavior of the field variables as a function of the spatial coordinates, the parameter vector and time.
\end{abstract}

Keywords: Reduced-order model; physics-based surrogate model; time-dependent parametrized partial differential equation; proper orthogonal decomposition; radial basis functions

\section{Introduction}

Parametrized partial differential equations (PDEs) arise in a number of important application areas, including design optimization, uncertainty analysis, optimal control and inverse parameter estimation. The computational cost associated with these applications can be exorbitant,

${ }^{*}$ Corresponding author: Computational Engineering and Design Group, School of Engineering Sciences, University of Southampton, Highfield, Southampton SO17 1BJ, United Kingdom, Tel: +44(0)2380598519, Email P.B.Nair@soton.ac.uk 
particularly when the underlying PDE model is required to be solved with high accuracy using a fine spatial mesh and small time-steps. In order to solve challenging problems on a limited computational budget, there is a need for efficient numerical methods for constructing approximation models (also commonly known as surrogates or emulators) of parametrized PDEs. Such techniques enable the PDE solution to be efficiently approximated at any point in the parameter space, thereby leading to significant computational cost savings in applications requiring multiple evaluations of the PDE solution over the parameter space of interest. This has motivated a number of researchers to investigate numerical methods for approximating the solution of parametrized PDEs.

Reduced-order modeling (ROM) has emerged as a powerful approach for tackling parametrized PDEs and a number of formulations based on this idea has been proposed in the literature. The basic idea underlying ROM is to approximate the solution using an appropriate set of basis vectors/functions and subsequently estimate the undetermined coefficients in the expansion using Galerkin projection or an error minimization scheme. Existing approaches include methods based on Lagrange, Hermite or Taylor subspace and proper orthogonal decomposition (POD) strategies; see references $[1,2,3]$ for an overview. However, most of the work on this topic has focused on parametrized steady-state PDEs $[4,5,6,7,8,9]$ and very little work has been done on developing general computational methods for ROM of time-dependent parametrized nonlinear PDEs. This can be primarily attributed to the inevitable computational difficulties that arise when it is sought to approximate the PDE solution as a function of the spatial coordinates, time and the parameter vector.

In [10], we proposed a principal component analysis (PCA) methodology to construct ROMs of steady-state parametrized PDEs. The key idea was to apply PCA to a training dataset obtained by solving the fine solver at a set of design points chosen using a design of computer experiments (DoCE) algorithm to derive a set of spatial and parameter-space basis functions. A greedy adaptive algorithm was developed to ensure that the method scales well to high-dimensional problems that may necessitate a large number of runs of the fine solver. Detailed numerical studies were presented to demonstrate that this approach allows for the construction of highly accurate ROMs with modest computational effort.

Time-dependent parametrized PDEs are much more challenging compared to steady-state problems, particularly when the boundary conditions vary as a function of time and the parameter space. The main difficulty arises from the requirement of constructing a reduced-order approximation model that satisfies the initial and boundary conditions at all points in the parameter space. Gunzburger et al. [11], studied this problem for a special class of parametrized PDE models, where only the boundary conditions are parametrized. However, this approach cannot be readily extended to problems where the governing equations are also parametrized. Hay et al. [12] proposed sensitivity-based approaches for constructing reduced-order models of unsteady PDEs over parametrized geometries. Both these approaches are based on Galerkin projection due 
to which they can be difficult to implement for problems with complicated nonlinearity terms. It is worth mentioning here that the reduced basis method studied in [13] has been applied to the unsteady Burgers' equation in one space dimension and a posteriori error bounds were derived for the approximation. The so-called discrete empirical interpolation method [14] is also a very interesting approach that has been applied for approximating the solution of nonlinear parametrized PDEs.

In this paper, we present a general non-intrusive method for constructing reduced-order approximations to the solution of time-dependent parametrized nonlinear PDEs, where the governing equations, the boundary and initial conditions are parametrized. The methodology presented here can be considered to be a generalization of our PCA based method for steady-state parametrized PDEs. Our goal is to construct a ROM that can eventually be used to approximate the PDE solution at a huge number of points in the parameter space very efficiently. The key idea underpinning the proposed method is to split the reduced-order approximation into two terms. The first term is defined as the solution of an auxiliary parabolic linear parametrized PDE - this is to guarantee that the ROM satisfies the boundary and initial conditions by construction. The second term in the approximation is composed of a linear combination of a tensor product of physical space and temporal domain empirical proper orthogonal modes. We propose a two-level POD approach for constructing the spatial and temporal basis functions starting from an ensemble of solution snapshots obtained by solving the original PDEs at a finite set of points in the parameter space. The undetermined coefficients in the approximation are estimated using a non-intrusive approach based on radial basis function approximation (in contrast to Galerkin projection), thereby enabling the straightforward application of our methodology to parametrized PDEs with complicated nonlinearity terms. We present numerical studies for a model parametrized Burgers' equation and a parametrized form of the convection-reactiondiffusion problem to illustrate the accuracy of the proposed approach.

The remainder of this paper is organized as follows: In Section 2, we outline the central ideas used in the proposed formulation. In the section that follows, we show how spatial and temporal basis functions that obey certain conditions can be constructed using a two-level POD method. Subsequently, in Section 4, we present a nonintrusive method based on radial basis function approximation to estimate the undetermined coefficients in the reduced-order approximation. Section 5 focuses on approximating the solution of the auxiliary parametrized parabolic PDE so that the ROM can be evaluated at any point in the parameter space in real-time. Section 6 is devoted to numerical studies for a parametrized Burgers' equation. Eventually, numerical results are presented in Section 7 for a more complex parametrized convection-reaction-diffusion problem. Section 8 concludes the paper and outlines some possible directions for further work on this topic. 


\section{Problem statement and methodology}

Consider the parametrized time-dependent PDE model given below:

$$
\frac{\partial u^{\theta}}{\partial t}+\mathscr{N}\left(u^{\theta}, \nabla u^{\theta}\right)=f^{\theta} \text { in } \Omega \times(0, T]
$$

where $\boldsymbol{\theta} \in] 0,1\left[^{p}(p \geq 1)\right.$ is a vector of $p$ parameters, $t \in(0, T]$ denotes time and $\Omega$ is the physical domain over which the PDE operator is defined with regular boundary $\partial \Omega$. We denote by $\mathscr{N}\left(u^{\theta}, \nabla u^{\theta}\right)$ a nonlinear parametrized operator; $u^{\theta}$ is the field variable which we seek to approximate as a function of the physical coordinates $\mathbf{x}$, the parameter vector $\boldsymbol{\theta}$ and time $t$. The governing equations are supplemented by parametrized boundary and initial conditions of the form

$$
\begin{aligned}
u^{\theta}(\cdot, t) & =g^{\theta}(\cdot, t) \text { on } \partial \Omega \times(0, T], \\
u_{\left.\right|_{t=0} ^{\theta}}^{\theta} & =u_{0}^{\theta}(\mathbf{x}) \text { in } \Omega .
\end{aligned}
$$

For any value of $\boldsymbol{\theta}$, it is assumed that (1-3) is well-posed in a usual Hilbert space made of regular functions - typically $H^{1}(\Omega) \otimes L^{2}(0, T)$ - meaning that $u_{0}^{\boldsymbol{\theta}}, f^{\boldsymbol{\theta}}$ and $g^{\boldsymbol{\theta}}$ are smooth enough related to the nonlinearity term $\mathscr{N}(\cdot)$.

Our objective is to construct a reduced-order approximation of the solution of (1-3) as a function of $\mathbf{x}, \boldsymbol{\theta}$ and $t$. One straightforward approach to tackle this problem would be to work with the finite-dimensional representation of (1-3) obtained after spatial discretization with mesh spacing $h$ and an appropriate time-stepping scheme. Then for each time instant of interest, say $t_{n}$, the field variable can be approximated as a function of space $\mathbf{x}$ and $\boldsymbol{\theta}$ using the methodology outlined in [10] for steady-state parametrized PDEs. Even though this approach is easy to implement, it is computationally not very attractive due to the need for constructing a ROM at each time step. We shall not pursue this approach any further and instead focus on developing numerical schemes that deal with time as a continuous variable.

In order to ensure that the approximation for $u^{\theta}(\mathbf{x}, t)$ satisfies the boundary and initial conditions for any value of $\boldsymbol{\theta}$, we propose an ansatz of the form:

$$
\widehat{u}^{\theta}(\mathbf{x}, t)=v^{\theta}(\mathbf{x}, t)+\sum_{k=1}^{K} \sum_{m=1}^{M} \alpha_{k m}(\boldsymbol{\theta}) \varphi^{k}(\mathbf{x}) \xi^{m}(t)
$$

where $\alpha_{k m}, k=1, \ldots, K, m=1, \ldots, M$ denote a set of undetermined coefficients in the approximation, and $\varphi^{k}(\mathbf{x})$ and $\xi^{m}(t)$ are spatial and temporal basis functions with the following properties:

$$
\begin{aligned}
& \varphi_{\mid \partial \Omega}^{k}=0, \forall k=1, \ldots, K, \\
& \xi^{m}(0)=0, \forall m=1, \ldots, M . \\
& 4
\end{aligned}
$$


The above conditions essentially state that all the spatial basis functions $\varphi^{k}$ are zero on the boundary $\partial \Omega$, while all the temporal basis functions $\xi^{m}$ are zero at $t=0$. Due to these properties of the spatial and temporal basis functions, we now only need to choose the term $v^{\theta}(\mathbf{x}, t)$ such that the parametrized boundary and initial conditions (2-3) are satisfied by construction. For the special case when the boundary conditions do not vary as a function of time, we can set the first term in (4) as $v^{\theta}(\mathbf{x}, t)=u_{0}^{\theta}(\mathbf{x})$. Due to the properties of our basis functions stated in (5), it can be easily seen that for $t=0$, we have

$$
\widehat{u}^{\theta}(\mathbf{x}, 0)=u_{0}^{\theta}(\mathbf{x}),
$$

and in addition

$$
\widehat{u}_{\mid \partial \Omega}^{\theta}=u_{0_{\partial \Omega \Omega}}^{\theta}=g^{\theta} .
$$

Therefore, it follows that for the above choice of $v^{\theta}(\mathbf{x}, t)$, the initial and boundary conditions are automatically satisfied by the approximation (4).

However, the choice for $v^{\theta}(\mathbf{x}, t)$ is not that obvious for the more general case when the boundary conditions are time-dependent. In this paper, we propose the idea that solution of the following auxiliary parabolic linear $\mathrm{PDE}^{1}$ can be chosen to be the first term in the approximation (4)

$$
\begin{aligned}
& \frac{\partial v^{\theta}}{\partial t}-\Delta v^{\theta}=0 \text { in } \Omega \times(0, T], \\
& v^{\theta}(\cdot, t)=g^{\theta}(\cdot, t) \text { on } \partial \Omega \times(0, T], \\
& v_{l_{t=0}^{\theta}}^{\theta}=u_{0}^{\theta} \text { in } \Omega .
\end{aligned}
$$

It can be clearly seen that due to the prescribed properties of the chosen spatial and temporal basis functions (5) and the above choice of $v^{\theta}$, our approximation will automatically satisfy both initial and boundary conditions.

In the next section, we shall delve into details of how basis functions with properties (5) can be constructed using a two-level POD method. After an appropriate set of basis functions have been constructed, the approximation problem eventually boils down to estimation of the undetermined coefficients $\alpha_{k m}$ in (4). A commonly used approach in ROM construction is the Galerkin method, wherein the approximation (4) is substituted into the original nonlinear parametrized equations (1-3) and the residual error is made orthogonal with respect to the approximating space of basis functions. This approach, however, is not straightforward to implement when the nonlinear parametrized term $\mathscr{N}\left(u^{\theta}, \nabla u^{\theta}\right)$ has a complicated structure [21]. In the present work, we propose a general nonintrusive approach that circumvents this difficulty. Subsequently, we outline the steps involved in numerical solution of the auxiliary PDE model to approximate the term $v^{\theta}$ so that the reduced-order approximation (4) can be evaluated in real-time at any point

\footnotetext{
${ }^{1}$ Note that this auxiliary PDE model is a linear initial boundary value heat equation with parametrized boundary and initial conditions.
} 
in the parameter space. We will also discuss in the next section the limitations of the proposed methodology, namely which parametrized PDE models can be correctly approximated with such an approach.

\section{Construction of spatial and temporal basis functions}

In this section, we focus on constructing the spatial and temporal basis functions, namely $\varphi^{k}(x)$ and $\xi^{m}(t)$, that satisfy (5). The key idea underpinning our approach is to employ a twolevel POD procedure on data obtained by solving the original PDEs (fine solver) at a set of points in the parameter space.

To illustrate, consider the following space-filling set of design points within the parameter space

$$
\mathscr{W}^{I}=\left\{\boldsymbol{\theta}_{i} \in\right] 0,1\left[{ }^{p}, i=1, \ldots, I\right\} .
$$

Such a space-filling set of design points can be obtained using Latin Hypercube sampling or minimum discrepancy sequences such as Sobol, Halton and Faure sequences [15, 16]. The fine solver (i.e., a high-fidelity solver for the original nonlinear PDEs) can be run at these $I$ points to generate a training dataset that is eventually used in a two-level POD procedure to construct the spatial and temporal basis functions that satisfy the conditions outlined earlier in (5).

Next, we introduce a coarse sampling of the spatial domain, i.e.,

$$
\mathscr{X}^{J}=\left\{\mathbf{x}_{j} \in \Omega_{h}, j=1, \ldots, J\right\},
$$

where $\Omega_{h}$ denotes the discretized computational domain with the index $h$ referring to the spatial mesh diameter. Similarly, a coarse sampling of the temporal domain can be written in the form

$$
\mathscr{Y}^{N}=\left\{t_{n}, 0=t_{1}<\cdots<t_{N}=T\right\} .
$$

Consider the following set of shifted snapshots obtained by running the fine solver (1-3) and the auxiliary PDE model (6-8) at a point (say $\boldsymbol{\theta}_{i}$ ) within the set (9):

$$
\mathscr{S}_{i}^{N}=\left\{u^{\theta_{i}}\left(\cdot, t_{n}\right)-v^{\theta_{i}}\left(\cdot, t_{n}\right), n=1, \ldots, N\right\} .
$$

Note that the shifted snapshots are computed at all time-instants defined in the coarse temporal domain sampling (11). It is also worth emphasizing that we use here a subscript $i$ for the snapshot set $\mathscr{S}_{i}^{N}$ to highlight the fact that we compute a set of shifted snapshots for each point in the set (9).

Consider the spatial Gram matrix defined below

$$
\left(\mathbf{M}_{\mathbf{x}}^{i}\right)_{n m}=\left(u^{\theta_{i}}\left(\cdot, t_{n}\right)-v^{\theta_{i}}\left(\cdot, t_{n}\right), u^{\theta_{i}}\left(\cdot, t_{m}\right)-v^{\theta_{i}}\left(\cdot, t_{m}\right)\right)_{L^{2}\left(\Omega_{h}\right)}, n, m=1, \ldots, N,
$$


where the $L^{2}$-scalar product is defined as

$$
(u, v)_{L^{2}\left(\Omega_{h}\right)}=\int_{\Omega_{h}} u(\mathbf{x}) v(\mathbf{x}) d \mathbf{x} .
$$

Let us denote by $\left(\lambda_{n}^{i}\right)_{n=1, \ldots, N}$ the positive eigenvalues of $\mathbf{M}_{\mathbf{x}}^{i}$ arranged in descending order

$$
\lambda_{1}^{i} \geq \lambda_{2}^{i} \geq \cdots \geq \lambda_{N}^{i} \geq 0
$$

We assume that the spectrum of the Gram matrix decays rapidly, which is typically the case for a large class of elliptic and parabolic PDEs. ${ }^{2}$ In other words, for a given small threshold $\varepsilon>0$, there exists an integer $K^{i}=K^{i}(\varepsilon)$ with $K^{i} / N$ small enough such that

$$
\frac{\sum_{k=1}^{K^{i}} \lambda_{k}^{i}}{\sum_{k=1}^{N} \lambda_{k}^{i}} \geq 1-\varepsilon
$$

meaning that the solutions of the considered PDE problems have a principal direction property. The first $K^{i}$ eigenfunctions $\left(\varphi^{k, i}\right)_{k}$ associated with the first $K^{i}$ eigenvalues $\lambda_{k}^{i}$, provide the orthogonal principal directions of the set $\mathscr{S}_{i}^{N}$. If $\mathbf{r}^{k, i}=\left(\mathbf{r}^{k, i}\right)_{n}$ denotes the $k$ th eigenvector of $\mathbf{M}_{\mathbf{x}}^{i}$, the $k$ th eigenfunction $\varphi^{k, i}$ can be computed as

$$
\varphi^{k, i}(\mathbf{x})=\sum_{n=1}^{N}\left(\mathbf{r}^{k, i}\right)_{n}\left(u^{\theta_{i}}\left(\mathbf{x}, t_{n}\right)-v^{\theta_{i}}\left(\mathbf{x}, t_{n}\right)\right)
$$

and then normed in the $L^{2}\left(\Omega_{h}\right)$-sense.

In summary, what we have done so far is to carry out PCA of the set of shifted snapshots $\mathscr{S}_{i}^{N}$, to compute a set of basis functions $\left(\varphi^{k, i}\right)_{k=1, \ldots, K^{i}}$, where $K^{i}$ is a (small) number. For more details concerning the POD method (also referred as Principal Component Analysis (PCA) or Karhunen-Loève decomposition), we refer the reader to [17, 18, 19].

After the basis sets $\left(\varphi^{k, i}\right)_{k=1, \ldots, K^{i}}$ have been computed for all parameter space points $\boldsymbol{\theta}_{i} \in \mathscr{W}^{I}$, we apply, as in [20], a second PCA to all the families of spatial modes previously computed, to construct a set of common POD modes $\left(\varphi^{k}\right)_{k=1, \ldots, K}$.

A similar two-level POD method can be employed to construct temporal basis functions $\left(\xi^{m}\right)_{m=1, \ldots, M}$. For each parameter space point $\boldsymbol{\theta}_{i} \in \mathscr{W}^{I}$ and $\mathbf{x}_{j} \in \mathscr{X}^{J}$, we first construct $M^{i}$ temporal POD modes $\left(\xi^{m, i}\right)_{m=1, \ldots, M^{i}}$, from the set

$$
\mathscr{T}_{i}^{J}=\left\{u^{\theta_{i}}\left(\mathbf{x}_{j}, \cdot\right)-u_{0}^{\theta_{i}}\left(\mathbf{x}_{j}\right), j=1, \ldots, J\right\}
$$

\footnotetext{
${ }^{2}$ We wish to highlight here that this assumption may not hold for hyperbolic problems where the Gram matrix spectrum decay can be very slow. In addition, hyperbolic problems often involve discontinuous solutions which are poorly approximated by POD-type methods.
} 
and then apply a second PCA on $\left(\xi^{m, i}\right)_{m, i}$ to get the temporal basis functions.

The following proposition holds:

Proposition 1. Let $\mathscr{S}_{i}^{N}$ (resp. $\mathscr{T}_{i}^{J}$ ) be the spatial (resp. temporal) snapshot sets given by (12) (resp. (14)). Then the spatial and temporal basis functions $\varphi^{k}$ and $\xi^{m}$ satisfy the properties (5), meaning that the ROM $\widehat{u}^{\theta}$ given by (4) satisfies both the boundary and initial conditions by construction.

Proof. To prove this result, we use the expansion (13) of $\varphi^{k, i}$ as a linear combination of shifted spatial snapshots, and exploit a property of the "snapshot method" developed by Sirovich [17]. By construction, since $u^{\theta_{i}}\left(\cdot, t_{n}\right)_{\mid \partial \Omega}=v^{\theta_{i}}\left(\cdot, t_{n}\right)_{\mid \alpha \Omega}=g^{\theta_{i}}\left(\cdot, t_{n}\right)$ we deduce that

$$
\varphi^{k, i}\left(\cdot, t_{n}\right)_{\mid \partial \Omega}=0, \forall k=1, \ldots K^{i} .
$$

Applying a PCA to all the basis sets $\left(\varphi^{k, i}\right)_{k, i}$ then leads to spatial modes $\left(\varphi^{k}\right)_{k=1, \ldots, K}$ that are also linear combinations of $\left(\varphi^{k, i}\right)_{k, i}$. Consequently the modes $\varphi^{k}$ also vanish on $\partial \Omega$.

Similar arguments can be used to establish the stated properties of the temporal basis functions $\xi^{m}$ since we have

$$
\xi^{m, i}(t)=\sum_{j=1}^{J}\left(\mathbf{s}^{m, i}\right)_{j}\left(u^{\boldsymbol{\theta}_{i}}\left(\mathbf{x}_{j}, t\right)-u_{0}^{\boldsymbol{\theta}_{i}}\left(\mathbf{x}_{j}\right)\right),
$$

where $\mathbf{s}^{m, i}=\left(\mathbf{s}^{m, i}\right)_{j}$ denotes the $m$ th eigenvector of the temporal Gram matrix $\mathbf{M}_{t}^{i}$ defined as

$$
\left(\mathbf{M}_{t}^{i}\right)_{j j^{\prime}}=\left(u^{\theta_{i}}\left(\mathbf{x}_{j}, \cdot\right)-u_{0}^{\theta_{i}}\left(\mathbf{x}_{j}\right), u^{\theta_{i}}\left(\mathbf{x}_{j^{\prime}}, \cdot\right)-u_{0}^{\theta_{i}}\left(\mathbf{x}_{j^{\prime}}\right)\right)_{L^{2}([0, T]}, j, j^{\prime}=1, \ldots, J .
$$

Since we have

$$
\xi^{m, i}(0)=0, \forall i=1, \ldots, I, \forall m=1, \ldots, M^{i}
$$

it follows that $\xi^{m}(0)=0, \forall m=1, \ldots, M$.

\section{Estimation of the undetermined ROM coefficients}

In this section, we look at how the undetermined coefficients $\alpha_{k m}$ of the ROM (4) can be computed for any design point $\boldsymbol{\theta}$. We proceed as follows. In the first step, we compute the coefficients $\alpha_{k m}$ for each design point $\boldsymbol{\theta}_{i} \in \mathscr{W}^{I}$. Considering the space-filling sets $\mathscr{X}^{J}$ and $\mathscr{Y}^{N}$, we have, for a fixed value of $\boldsymbol{\theta}_{i}$,

$$
\widehat{u}^{\boldsymbol{\theta}_{i}}\left(\mathbf{x}_{j}, t_{n}\right)=v^{\boldsymbol{\theta}_{i}}\left(\mathbf{x}_{j}, t_{n}\right)+\sum_{k=1}^{K} \sum_{m=1}^{M} \alpha_{k m}\left(\boldsymbol{\theta}_{i}\right) \varphi^{k}\left(\mathbf{x}_{j}\right) \xi^{m}\left(t_{n}\right),
$$

$\forall j=1, \ldots, J, \forall n=1, \ldots, N$. For a fixed index $i$, the preceding equation can be written in the compact form

$$
\widehat{\mathbf{u}}^{i}=\mathbf{v}^{i}+\varphi \alpha^{i} \xi^{T}
$$


where $\widehat{\mathbf{u}}^{i} \in \mathscr{M}_{J N}$ with coefficients $\left(\widehat{\mathbf{u}}^{i}\right)_{j n}=\widehat{u}^{\theta_{i}}\left(\mathbf{x}_{j}, t_{n}\right), \mathbf{v}^{i} \in \mathscr{M}_{J N}$ with coefficients $\left(\mathbf{v}^{i}\right)_{j n}=$ $v^{\theta_{i}}\left(\mathbf{x}_{j}, t_{n}\right), \varphi \in \mathscr{M}_{J K}$ with coefficients $(\varphi)_{j k}=\varphi^{k}\left(\mathbf{x}_{j}\right), \boldsymbol{\alpha}^{i} \in \mathscr{M}_{K M}$ with coefficients $\left(\boldsymbol{\alpha}^{i}\right)_{k m}=$ $\alpha_{k m}\left(\boldsymbol{\theta}_{i}\right)$, and $\boldsymbol{\xi} \in \mathscr{M}_{N M}$ with coefficients $(\boldsymbol{\xi})_{n m}=\xi^{m}\left(t_{n}\right)$.

The undetermined coefficients $\boldsymbol{\alpha}^{i}$ can be computed by solving

$$
\mathbf{u}^{i}=\mathbf{v}^{i}+\varphi \alpha^{i} \xi^{T}
$$

where $\mathbf{u}^{i} \in \mathscr{M}_{J N}$ denote the extracted elements of the fine solutions, that is to say $\left(\mathbf{u}^{i}\right)_{j n}=$ $u^{\theta_{i}}\left(\mathbf{x}_{j}, t_{n}\right)$. Since the columns of $\varphi$ and $\xi$ are orthonormal, we deduce the coefficients $\boldsymbol{\alpha}^{i}$ by the relations

$$
\boldsymbol{\alpha}^{i}=\varphi^{T}\left(\mathbf{u}^{i}-\mathbf{v}^{i}\right) \xi
$$

The final step involves approximating the coefficients $\alpha_{k m}(\boldsymbol{\theta})$ as a function of $\boldsymbol{\theta}$ so that they can be evaluated at any arbitrary point in the parameter space efficiently. For fixed $k, m$, we first expand the undetermined coefficients using Radial Basis Functions (RBFs) as follows

$$
\alpha_{k m}(\boldsymbol{\theta})=\sum_{i=1}^{I} \gamma_{i}^{k m} \Phi\left(\frac{\left|\boldsymbol{\theta}-\boldsymbol{\theta}_{i}\right|}{\sigma}\right) .
$$

Setting $\boldsymbol{\theta}=\boldsymbol{\theta}_{i^{\prime}}$ in (20) for $i^{\prime}=1, \ldots, I$, leads to

$$
\sum_{i=1}^{I} \mathbf{A}_{i^{\prime}} \gamma_{i}^{k m}=\alpha_{k m}\left(\boldsymbol{\theta}_{i^{\prime}}\right)
$$

where the symmetric interpolation matrix $\mathbf{A}$ is such that $\mathbf{A}_{i^{\prime} i}=\Phi\left(\frac{\left|\boldsymbol{\theta}_{i^{\prime}}-\boldsymbol{\theta}_{i}\right|}{\sigma}\right)$. We give more details about the choice of $\Phi$ and $\sigma$ later in Section 7.3. The preceding equation can be rewritten in compact form as

$$
\mathbf{A} \boldsymbol{\gamma}^{k m}=\mathbf{b}^{k m},
$$

where $\mathbf{b}^{k m}$ is a vector of length $I$ defined by $\left(\mathbf{b}^{k m}\right)_{i}=\alpha_{k m}\left(\boldsymbol{\theta}_{i}\right)$. The unknown coefficients $\boldsymbol{\gamma}^{k m}$ can be calculated by solving the following penalized normal equations (again with a small regularization parameter $\mu>0$ )

$$
\left(\mathbf{A}^{T} \mathbf{A}+\mu I\right) \boldsymbol{\gamma}^{k m}=\mathbf{A}^{T} \mathbf{b}^{k m} .
$$

Given the solution of the above matrix system of equations, the undetermined coefficients can be efficiently computed at any point in the parameter space using (20).

\section{Enabling real-time predictions using the ROM}

\subsection{Motivation}

We now look at how the ROM (4) can be employed in a real-time prediction framework. There are essentially two options available to the user of such a ROM, depending on the timedependent PDE model which is under consideration. 
In the first case, let us consider the scenario when the ROM is to be evaluated at a limited number of points in the parameter space. Then we can directly use the expansion (4) to get approximate solutions $\widehat{u}^{\theta}$ for different values of $\boldsymbol{\theta}$. Once the spatial and temporal modes $\varphi^{k}$ and $\xi^{m}$ have been computed through the two-level PCA procedures (see Section 3), we can compute the coefficients $\alpha_{k m}(\boldsymbol{\theta})$ using the methodology described in Section 4: use (19), next solve (23) and then use (20). To compute $\widehat{u}^{\theta}$, the final step involves computing $v^{\theta}$ (i.e., the solution of the auxiliary parabolic linear PDE (6-8)).

The second case involves the scenario where it is required to compute $\widehat{u}^{\theta}$ at a large number of points in the parameter space. In principle, the steps outlined earlier can still be employed; however, the main computational obstacle arises from computation of the term $v^{\theta}$ while making predictions at any point in the parameter space. This is because direct numerical simulations of (6-8) cannot be done efficiently (say in real-time), even though the auxiliary PDE model is linear. Consequently one needs to construct an adapted ROM for (6-8), which is not an obvious task since the boundary conditions are time-dependent. This would enable faster online evaluations of the reduced-order model at the expense of additional offline computations (due to the step of constructing an approximation model to enable efficient evaluation of the term $v^{\theta}$ ).

In this section we focus on efficient numerical solution of the auxiliary parabolic PDE (6-8). We take advantage of the linear nature of this equation to separate this PDE into two simpler ones: an initial value problem with homogeneous boundary conditions and a boundary value problem with zero initial conditions. The solution $v^{\theta}$ of (6-8) can be split according to

$$
v^{\theta}=w^{\theta}+z^{\theta}
$$

where $w^{\theta}$ and $z^{\theta}$ are, respectively, solutions of the following PDEs

$$
\begin{aligned}
& \frac{\partial w^{\theta}}{\partial t}-\Delta w^{\theta}=0 \text { in } \Omega \times(0, T], \\
& w^{\theta}(\cdot, t)=0 \text { on } \partial \Omega \times(0, T], \\
& w_{\mathrm{l} t=0}^{\theta}=u_{0}^{\theta} \text { in } \Omega,
\end{aligned}
$$

and

$$
\begin{aligned}
& \frac{\partial z^{\theta}}{\partial t}-\Delta z^{\theta}=0 \text { in } \Omega \times(0, T], \\
& z^{\theta}(\cdot, t)=g^{\theta}(\cdot, t) \text { on } \partial \Omega \times(0, T], \\
& z_{t=0}^{\theta}=0 \text { in } \Omega .
\end{aligned}
$$

Note that the first PDE (25-27) is a linear heat equation with homogeneous boundary conditions, while the second PDE (28-30) is a linear heat equation with zero initial conditions. We now move on to how the component terms $w^{\theta}$ and $z^{\theta}$ can be efficiently computed. 


\subsection{Resolution of the auxiliary PDE with homogeneous $B C$}

In order to approximate the solutions of (25-27) by a low-order model, we can use a classical POD-Galerkin approach provided the initial condition $u_{0}^{\theta}$ is smooth enough. In other words, the term $w^{\theta}$, which is parametrized through the initial value, is approximated as

$$
w^{\theta}(\mathbf{x}, t)=\sum_{k=1}^{K_{I V}} a_{k}^{\theta}(t) \varphi_{I V}^{k}(\mathbf{x}),
$$

where $a_{k}^{\boldsymbol{\theta}}(t)$ denote a set of undetermined coefficients which is an implicit function of $\boldsymbol{\theta}$. We denote by $\varphi_{I V}^{k}(\mathbf{x})$ a set of basis functions obtained via the POD method, i.e., by applying PCA to the following snapshot dataset

$$
\left\{w^{\boldsymbol{\theta}_{i}}\left(\cdot, t_{n}\right), i=1, \ldots, I, n=1, \ldots, N\right\} .
$$

Now, for a fixed value of the parameter vector $\boldsymbol{\theta}$, the undetermined coefficients in (31) can be computed by solving the following (small) system of coupled linear ordinary differential equations

$$
\left\{\begin{array}{l}
\frac{d \mathbf{a}^{\boldsymbol{\theta}}}{d t}+\mathbf{R} \mathbf{a}^{\boldsymbol{\theta}}=0, \\
\mathbf{a}_{k}^{\boldsymbol{\theta}}(0)=\left(u_{0}^{\boldsymbol{\theta}}, \varphi_{I V}^{k}\right)_{L^{2}\left(\Omega_{h}\right)}
\end{array}\right.
$$

where the stiffness matrix is defined as

$$
\mathbf{R}_{k l}=\int_{\Omega_{h}} \nabla \varphi_{I V}^{k}(\mathbf{x}) \nabla \varphi_{I V}^{l}(\mathbf{x}) d \mathbf{x}
$$

and $\mathbf{a}^{\theta}=\left(a_{1}^{\theta}, \ldots, a_{K_{I V}}\right)^{T}$.

It is worth noting here that since the stiffness matrix is not a function of $\boldsymbol{\theta}$, it can be precomputed once for all. Hence, the solution of (33) can be computed efficiently for a given value of $\boldsymbol{\theta}$. Given the solution of (33), the term $w^{\boldsymbol{\theta}}(\mathbf{x}, t)$ can be computed for any arbitrary value of $\boldsymbol{\theta}$ using (31).

\subsection{Resolution of the auxiliary PDE with zero initial condition}

We need to exercise particular care while solving (28-30) since the boundary function $g^{\theta}(\cdot, t)$ is time-dependent. Once again, we take advantage of the linear feature of this PDE. A POD Petrov-Galerkin projection scheme appears to be suited in this case. To begin with, let us consider a set of boundary conditions $L^{2}(\partial \Omega)$-valued snapshots

$$
\mathscr{U}_{n}^{I}=\left\{g^{\theta_{i}}\left(\cdot, t_{n}\right), i=1, \ldots, I\right\},
$$

where $t_{n}$ belongs to a coarse temporal discretization. 
The idea behind using the set (34) is to identify the principal components related to the $\mathrm{BC}$ and to lower the dimension of the representative space of the time-dependent BC. Once a PCA is performed on the set $\mathscr{U}_{n}^{I}$, the first $K_{B C}$ POD modes $\eta^{k}\left(\cdot, t_{n}\right) \in L^{2}(\partial \Omega)$ associated with $\mathscr{U}_{n}^{I}$ are stored. This procedure is performed for all coarse time instants $t_{n}, n=1, \ldots, N$. From a computational point of view, the previous procedure is achievable since the $N$ PCA calculations can be run independently of each other in parallel. Moreover, the storage requirements of the corresponding $N K_{B C}$ modes $\eta^{k}$ is reasonable since $K_{B C}$ is expected to be small, $N$ is not too large since it corresponds to the size of a coarse temporal grid, and also because $\eta^{k}$ are $L^{2}(\partial \Omega)$-valued functions.

For a fixed instant time $t$, let us now denote by $\pi g^{\theta}$ the projection of the trace function $g^{\theta}(\cdot, t)$ onto the linear vector space spanned by the family $\left\{\eta^{1}(\cdot, t), \ldots, \eta^{K_{B C}}(\cdot, t)\right\}$ :

$$
\pi g^{\theta}(\cdot, t)=\sum_{k=1}^{K_{B C}} c_{k}^{\theta}(t) \eta^{k}(\cdot, t)
$$

with

$$
c_{k}^{\theta}(t)=\left(g^{\theta}(\cdot, t), \eta^{k}(\cdot, t)\right)_{L^{2}(\partial \Omega)} .
$$

Next, we then define the function $\tilde{z}^{\theta}$

$$
\tilde{z}^{\theta}(\mathbf{x}, t)=\sum_{k=1}^{K_{B C}} c_{k}^{\theta}(t) \xi^{k}(\mathbf{x}, t),
$$

where $\left\{\xi^{1}(\cdot, t), \ldots, \xi^{K_{B C}}(\cdot, t)\right\}$ are the solutions of the following $K_{B C}$ secondary equations that are independent of $\boldsymbol{\theta}$ :

$$
\begin{aligned}
& \frac{\partial \xi^{k}}{\partial t}-\Delta \xi^{k}=0 \text { in } \Omega \times(0, T], \\
& \xi^{k}(\cdot, t)=\eta^{k}(\cdot, t) \text { on } \partial \Omega \times(0, T], \\
& \xi_{t=0}^{k}=0 \text { in } \Omega,
\end{aligned}
$$

for $k=1, \ldots, K_{B C}$. The following proposition holds:

Proposition 2. Let $\tilde{z}^{\theta}$ be defined by (36-37) and $\left(\xi^{k}\right)$ by (38-40). Then $\tilde{z}^{\theta}$ is the solution of the problem

$$
\begin{aligned}
& \frac{\partial \tilde{z}^{\theta}}{\partial t}-\Delta \tilde{z}^{\theta}=\sum_{k=1}^{K_{B C}} \dot{c}_{k}^{\theta}(t) \xi^{k} \text { in } \Omega \times(0, T], \\
& \tilde{z}^{\theta}(\cdot, t)=\pi g^{\theta}(\cdot, t) \text { on } \partial \Omega \times(0, T], \\
& \tilde{z}_{t=0}^{\theta}=0 \text { in } \Omega .
\end{aligned}
$$


Proof. In $\Omega \times(0, T]$ we have, from (37):

$$
\begin{aligned}
\frac{\partial \tilde{z}^{\theta}}{\partial t}-\Delta \tilde{z}^{\theta} & =\sum_{k=1}^{K_{B C}} c_{k}^{\theta}(t)\left(\frac{\partial \xi^{k}}{\partial t}-\Delta \xi^{k}\right)+\sum_{k=1}^{K_{B C}} \dot{c}_{k}^{\theta}(t) \xi^{k} \\
& =\sum_{k=1}^{K_{B C}} \dot{c}_{k}^{\theta}(t) \xi^{k} \text { from (38). }
\end{aligned}
$$

Eq. (42) directly follows from (39):

$$
\tilde{z}_{\mid \partial \Omega}^{\boldsymbol{\theta}}=\sum_{k=1}^{K_{B C}} c_{k}^{\boldsymbol{\theta}}(t) \xi_{\left.\right|_{\partial \Omega}}^{k}=\sum_{k=1}^{K_{B C}} c_{k}^{\boldsymbol{\theta}}(t) \eta^{k}=\pi g^{\boldsymbol{\theta}}
$$

and eq. (43) from (40):

$$
\tilde{z}_{\left.\right|_{t=0}}^{\theta}=\sum_{k=1}^{K_{B C}} c_{k}^{\theta}(0) \xi_{\left.\right|_{t=0} ^{k}}^{k}=0
$$

In our ROM, we propose to use $\tilde{z}^{\theta}$ defined by (37) as an approximation for $z^{\theta}$, the solution of the original equation (28-30). We theoretically justify this approximation later in Section 5.4, where we provide an upper bound for $\left\|\left(z^{\theta}-\tilde{z}^{\theta}\right)(\cdot, t)\right\|_{L^{2}(\Omega)}$.

We now discuss different computational aspects of such a methodology, showing that the computations at every step can be carried out efficiently. First of all, it has to be noted that both $\eta^{k}$ and $\xi^{k}$ have to be known on the fine temporal grid because of (37) and (39). However, it seems impossible to directly compute $\eta^{k}$ on the fine temporal grid since it would require us to perform a PCA for each time instant which would be computationally prohibitive. Therefore we propose the following strategy: once $\eta^{k}$ are computed on a coarse temporal grid as described previously, we deduce its values on a fine grid using temporal interpolations. More precisely, for any point $\mathbf{x}_{j} \in \mathscr{X}^{J} \cap \partial \Omega_{h}$ we interpolate the set of values $\left\{y^{k j}=\eta^{k}\left(\mathbf{x}_{j}, t_{n}\right), n=1 \ldots, N\right\}$, using classical one-dimension interpolation functions (such as linear or cubic spline interpolators).

Once the $\eta^{k}$ are known on the fine temporal grid, we have to solve the problems (38-40). These problems can be solved in parallel for each $\xi^{k}$ since they are independent of each other. Moreover, since these problems do not depend on $\boldsymbol{\theta}$, the dual basis $\left\{\xi^{1}, \ldots, \xi^{K_{B C}}\right\}$ can be precomputed once and for all and used as the low-order basis to approximate the solutions of (28-30).

We next examine an important aspect of our methodology, related to data storage complexity. From a computational point of view, it is not always feasible to keep in memory $K_{B C}$ spatiotemporal modes $\xi^{k}$ that are needed to expand the solution $\tilde{z}^{\theta}$ (see (37)). To overcome this difficulty, we propose a second-level ROM with low-dimensional representation by projecting $\xi^{k}$ onto low-order spatial/temporal POD modes, for example:

$$
\xi^{k}(\mathbf{x}, t)=\sum_{l=1}^{L^{k}} \sum_{m=13}^{M^{k}} \beta_{l m}^{k} \chi^{k, l}(\mathbf{x}) \varsigma^{k, m}(t) .
$$


Using the second-level ROM (44) allows us to store $K_{B C}\left(L^{k} M^{k}+L^{k}\left(N_{x}\right)^{d}+M^{k} N_{t}\right)$ values, instead of $K_{B C}\left(N_{x}\right)^{d} N_{t}$ ones if we directly store the $\xi^{k}$ values on the fine spatial/temporal grids (respectively of size $\left(N_{x}\right)^{d}$ and $N_{t}$ ). Both $\left(\chi^{k, l}\right)_{l}$ and $\left(\varsigma^{k, m}\right)_{m}$ can be obtained by applying PCA to $\xi^{k}(\mathbf{x}, t)$ using a standard snapshot method for dataset generation. For $\mathbf{x}_{j}$ and $t_{n}$ belonging to the coarse spatial/temporal discretizations (10) and (11), we write, for $k=1, \ldots, K_{B C}$

$$
\xi^{k}\left(\mathbf{x}_{j}, t_{n}\right)=\sum_{l=1}^{L^{k}} \sum_{m=1}^{M^{k}} \beta_{l m}^{k} \chi^{k, l}\left(\mathbf{x}_{j}\right) \varsigma^{k, m}\left(t_{n}\right)
$$

The preceding equation can be rewritten in the compact form

$$
\boldsymbol{\xi}^{k}=\chi^{k} \boldsymbol{\beta}^{k}\left(\boldsymbol{\varsigma}^{k}\right)^{T}
$$

where we denote $\xi^{k} \in \mathscr{M}_{J N}$ with coefficients $\left(\xi^{k}\right)_{j n}=\xi^{k}\left(\mathbf{x}_{j}, t_{n}\right), \chi^{k} \in \mathscr{M}_{J L^{k}}$ with coefficients $\left(\chi^{k}\right)_{j l}=\chi^{k, l}\left(\mathbf{x}_{j}\right), \boldsymbol{\beta}^{k} \in \mathscr{M}_{L^{k} M^{k}}$ with coefficients $\left(\boldsymbol{\beta}^{k}\right)_{l m}=\beta_{l m}^{k}$ and $\boldsymbol{\varsigma}^{k} \in \mathscr{M}_{N M^{k}}$ with coefficients $\left(\boldsymbol{\varsigma}^{k}\right)_{n m}=\varsigma^{k, m}\left(t_{n}\right)$. Solving (45) with the penalized minimization problems

$$
\min _{\boldsymbol{\beta}^{k} \in \mathscr{M}_{L^{k} M^{k}}}\left\|\boldsymbol{\xi}^{k}-\chi^{k} \boldsymbol{\beta}^{k}\left(\boldsymbol{\varsigma}^{k}\right)^{T}\right\|^{2}+\mu\left\|\boldsymbol{\beta}^{k}\right\|^{2}
$$

where $\mu>0$ is a small regularization parameter, leads to the Euler-Lagrange equations

$$
\left(\left(\chi^{k}\right)^{T} \chi^{k}\right) \boldsymbol{\beta}^{k}\left(\left(\boldsymbol{\zeta}^{k}\right)^{T} \boldsymbol{\varsigma}^{k}\right)+\mu \boldsymbol{\beta}^{k}=\left(\chi^{k}\right)^{T} \boldsymbol{\xi}^{k} \boldsymbol{\zeta}^{k}
$$

We can reshape (47) as a linear algebraic system of equations size $L^{k} \times M^{k}$ as shown below

$$
\left(\mathbf{D}^{k}+\mu \mathbf{I}\right) \underline{\boldsymbol{\beta}}^{k}=\underline{\mathbf{d}}^{k}
$$

where

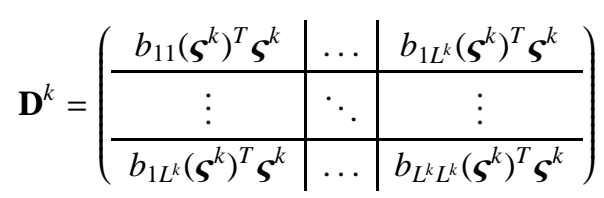

with $b_{i j}=\left(\left(\chi^{k}\right)^{T} \chi^{k}\right)_{i j}$, and where the rows of $\boldsymbol{\beta}^{k}$ (resp. of the rhs of (47)) are put in the vector $\underline{\beta}^{k}$ (resp. in $\underline{\mathbf{d}}^{k}$ ). Recall that a similar approach was previously used in the first-level ROM to estimate the undetermined coefficients $\alpha_{k m}(\boldsymbol{\theta})$; see Section 4.

\subsection{Error estimation of the auxiliary problem with zero initial condition}

We give here an error estimate between $z^{\theta}$ (i.e., the solution of (41-43)) and $z^{\theta}$ (i.e., the solution of the original equation (28-30)).

Proposition 3. Let $\Omega$ be a bounded subset of $\mathbb{R}^{d}$. Let $g^{\theta}$ be a boundary function such that $g^{\theta}$ and $\left.\frac{\partial g^{\theta}}{\partial t} \in C^{0}\left([0, T] ; L^{2}(\partial \Omega)\right), \forall \boldsymbol{\forall} \in\right] 0,1\left[{ }^{p}\right.$. If $z^{\theta}$ is the solution of (28-30) and $\tilde{z}^{\theta}$ is the solution of (41-43), then the following error estimate holds:

$$
\left\|\left(z^{\theta}-\tilde{z}^{\theta}\right)(\cdot, t)\right\|_{L^{2}(\Omega)} \leq A(t)+t B \exp \left(-\frac{t}{2 C_{p}(\Omega)^{2}}\right)
$$


where $C_{p}(\Omega)>0$ is the Poincaré constant, $A(t)$ is a function of time which can be arbitrary small depending on $K_{B C}, c_{k}^{\theta}$ and $\xi^{k}$, and $B$ is a constant depending on $\dot{c}_{k}^{\theta}, \xi^{k}$ and $T$.

Proof. As a first step, we give the weak formulation of (28-30). Because of its nonhomogeneous $\mathrm{BC}$, we formally define $y^{\boldsymbol{\theta}}=z^{\boldsymbol{\theta}}-g_{\Omega}^{\boldsymbol{\theta}}$ with $g_{\Omega}^{\boldsymbol{\theta}}=\sum_{k \geq 1} c_{k}^{\boldsymbol{\theta}} \xi^{k}$. It is easy to check that $g_{\Omega}^{\boldsymbol{\theta}}$ is an extension of $g^{\theta}$ to the whole domain $\Omega$, and that $y^{\theta}$ is the solution of

$$
\begin{aligned}
& \frac{\partial y^{\theta}}{\partial t}-\Delta y^{\theta}=b^{\theta} \text { in } \Omega \times(0, T], \\
& y^{\theta}(\cdot, t)=0 \text { on } \partial \Omega \times(0, T], \\
& y_{t=0}^{\theta}=0 \text { in } \Omega,
\end{aligned}
$$

with $b^{\theta}=\Delta g_{\Omega}^{\theta}-\frac{\partial g_{\Omega}^{\theta}}{\partial t}$. The weak form of (51-53) then reads

$$
\frac{d}{d t}\left(y^{\theta}, \varphi\right)_{L^{2}(\Omega)}+\left(\nabla y^{\theta}, \nabla \varphi\right)_{L^{2}(\Omega)}=\left(b^{\theta}, \varphi\right)_{L^{2}(\Omega)}, \forall \varphi \in H_{0}^{1}(\Omega) .
$$

We follow the same procedure for the problem (41-43). We define $\tilde{y}^{\theta}=\tilde{z}^{\theta}-\left(\pi g^{\theta}\right)_{\Omega}$ where $\left(\pi g^{\theta}\right)_{\Omega}=\sum_{k=1}^{K_{B C}} c_{k}^{\theta} \xi^{k}$ is the extension of $\pi g^{\theta}$ to the whole domain $\Omega$, so that $\tilde{y}^{\theta}$ is the solution of

$$
\begin{aligned}
& \frac{\partial \tilde{y}^{\theta}}{\partial t}-\Delta \tilde{y}^{\theta}=0 \text { in } \Omega \times(0, T], \\
& \tilde{y}^{\theta}(\cdot, t)=0 \text { on } \partial \Omega \times(0, T], \\
& \tilde{y}_{t=0}^{\theta}=0 \text { in } \Omega .
\end{aligned}
$$

The weak form of (55-57) is given by

$$
\frac{d}{d t}\left(\tilde{y}^{\theta}, \varphi\right)_{L^{2}(\Omega)}+\left(\nabla \tilde{y}^{\theta}, \nabla \varphi\right)_{L^{2}(\Omega)}=0, \forall \varphi \in H_{0}^{1}(\Omega) .
$$

Subtracting (58) to (54), and taking $\varphi=z^{\theta}-\tilde{z}^{\theta}$ as a test function, leads to

$$
\frac{d}{d t}\left(\left\|\left(y^{\theta}-\tilde{y}^{\theta}\right)(\cdot, t)\right\|_{L^{2}(\Omega)}^{2}\right)+\left\|\left(y^{\theta}-\tilde{y}^{\theta}\right)(\cdot, t)\right\|_{H_{0}^{1}(\Omega)}^{2}=\left(b^{\theta}, y^{\theta}-\tilde{y}^{\theta}\right)_{L^{2}(\Omega)} .
$$

Using Poincaré and Cauchy-Schwarz's inequalities (see [23, 24]) then gives

$$
\frac{d}{d t}\left(\left\|\left(y^{\theta}-\tilde{y}^{\theta}\right)(\cdot, t)\right\|_{L^{2}(\Omega)}\right)+\frac{1}{2 C_{p}(\Omega)^{2}}\left\|\left(y^{\theta}-\tilde{y}^{\theta}\right)(\cdot, t)\right\|_{L^{2}(\Omega)} \leq \frac{1}{2}\left\|b^{\theta}\right\|_{L^{2}(\Omega)}
$$

or, integrating over $[0, t]$ :

$$
\left\|\left(y^{\theta}-\tilde{y}^{\theta}\right)(\cdot, t)\right\|_{L^{2}(\Omega)} \leq \frac{1}{2} \int_{0}^{t}\left\|b^{\theta}(\cdot, s)\right\|_{L^{2}(\Omega)} d s-\frac{1}{2 C_{p}(\Omega)^{2}} \int_{0}^{t}\left\|\left(y^{\theta}-\tilde{y}^{\theta}\right)(\cdot, s)\right\|_{L^{2}(\Omega)} d s .
$$


Using the integral form of the Grönwall lemma (see [25]), we have the following inequality

$$
\left\|\left(y^{\theta}-\tilde{y}^{\theta}\right)(\cdot, t)\right\|_{L^{2}(\Omega)} \leq \alpha(t)-\frac{1}{2 C_{p}(\Omega)^{2}} \int_{0}^{t} \alpha(s) \exp \left(-\frac{t-s}{2 C_{p}(\Omega)^{2}}\right) d s
$$

where $\alpha(t)=\frac{1}{2} \int_{0}^{t}\left\|b^{\theta}(\cdot, s)\right\|_{L^{2}(\Omega)} d s$. Since $\alpha$ is a positive and nondecreasing function, we get

$$
\left\|\left(y^{\theta}-\tilde{y}^{\theta}\right)(\cdot, t)\right\|_{L^{2}(\Omega)} \leq \alpha(t) \exp \left(-\frac{t}{2 C_{p}(\Omega)^{2}}\right) .
$$

Coming back to the definition of $y^{\theta}$ and $\tilde{y}^{\theta}$, we have

$$
y^{\theta}-\tilde{y}^{\theta}=z^{\theta}-\tilde{z}^{\theta}-\sum_{k>K_{B C}} c_{k}^{\theta} \xi^{k}
$$

which yields

$$
\left\|\left(z^{\theta}-\tilde{z}^{\theta}\right)(\cdot, t)\right\|_{L^{2}(\Omega)} \leq\left\|\sum_{k>K_{B C}} c_{k}^{\theta}(t) \xi^{k}(\cdot, t)\right\|_{L^{2}(\Omega)}+\alpha(t) \exp \left(-\frac{t}{2 C_{p}(\Omega)^{2}}\right) .
$$

We then estimate $A(t)=\left\|\sum_{k>K_{B C}} c_{k}^{\theta}(t) \xi^{k}(\cdot, t)\right\|_{L^{2}(\Omega)}$. For this, we invoke the maximum principle property related to the heat problem (41-43). Since $g^{\theta_{i}} \in C^{0}\left([0, T] ; L^{2}(\partial \Omega)\right)$ for all $\boldsymbol{\theta}_{i}$, we deduce that $\eta^{k}$ also belong to $C^{0}\left([0, T] ; L^{2}(\partial \Omega)\right), \forall k=1, \ldots, K_{B C}$, by linearity of the POD modes with respect to the snapshots. As a consequence, we get from (35) that $\pi g^{\theta} \in$ $C^{0}\left([0, T] ; L^{2}(\partial \Omega)\right), \forall K_{B C}$. By virtue of the maximum principle, the solution $\tilde{z}^{\theta}$ of (41-43) belongs to $C^{0}\left([0, T] ; L^{2}(\Omega)\right), \forall K_{B C}$. It follows that the series $\left\|\sum_{k \geq 1} c_{k}^{\theta}(t) \xi^{k}(\cdot, t)\right\|_{L^{2}(\Omega)}$ converges, meaning that $A(t)$ can be arbitrary small provided that $K_{B C}$ is large enough.

The last step involves estimating the function $\alpha(t)$ which appears in (59). By construction, we have

$$
b^{\boldsymbol{\theta}}=\Delta g_{\Omega}^{\boldsymbol{\theta}}-\frac{\partial g_{\Omega}^{\boldsymbol{\theta}}}{\partial t}=\sum_{k \geq 1} c_{k}^{\theta}\left(\Delta \xi^{k}-\frac{\partial \xi^{k}}{\partial t}\right)-\sum_{k \geq 1} \dot{c}_{k}^{\theta} \dot{\xi}^{k}=-\sum_{k \geq 1} \dot{c}_{k}^{\theta} \dot{\xi}^{k}
$$

which leads to

$$
\alpha(t)=\frac{1}{2} \int_{0}^{t}\left\|b^{\theta}(\cdot, s)\right\|_{L^{2}(\Omega)} d s \leq t B
$$

with

$$
B=\frac{1}{2} \sup _{s \in[0, T]}\left\|\sum_{k \geq 1} \dot{c}_{k}^{\theta}(s) \xi^{k}(\cdot, s)\right\|_{L^{2}(\Omega)} .
$$

We justify the convergence of the series in (60) as follows. Using the Cauchy-Schwarz's inequality, we have, $\forall K_{B C}$ :

$$
\left\|\sum_{k=1}^{K_{B C}} \dot{c}_{k}^{\theta}(s) \xi^{k}(\cdot, s)\right\|_{L^{2}(\Omega)}^{2} \leq \sum_{k, l=1}^{K_{B C}} \mid \dot{c}_{k}^{\theta}(s)\left\|\dot{c}_{l}^{\theta}(s)\right\| \xi^{k}(\cdot, s)\left\|_{L^{2}(\Omega)}\right\| \xi^{l}(\cdot, s) \|_{L^{2}(\Omega)} .
$$


We first prove that

$$
\sup _{s \in[0, T]}\left\|\xi^{k}(\cdot, s)\right\|_{L^{2}(\Omega)}<+\infty, \forall k=1, \ldots, K_{B C} .
$$

Since $\eta^{k} \in C^{0}\left([0, T] ; L^{2}(\partial \Omega)\right)$, we deduce from the maximum principle applied to (38-40) that $\xi^{k} \in C^{0}\left([0, T] ; L^{2}(\Omega)\right)$, meaning that $(62)$ holds. We then need to show that

$$
\sup _{s \in[0, T]}\left|\dot{c}_{k}^{\theta}(s)\right|<+\infty, \forall k=1, \ldots, K_{B C} .
$$

Since $c_{k}^{\theta}(s)=\left(g^{\theta}(\cdot, s), \eta^{k}(\cdot, s)\right)_{L^{2}(\partial \Omega)}$ by definition, using the Cauchy-Schwarz's inequality, we have

$$
\left|\dot{c}_{k}^{\theta}(s)\right| \leq\left\|\frac{\partial g^{\theta}}{\partial t}(\cdot, s)\right\|_{L^{2}(\partial \Omega)}\left\|\eta^{k}(\cdot, s)\right\|_{L^{2}(\partial \Omega)}+\left\|g^{\theta}(\cdot, s)\right\|_{L^{2}(\partial \Omega)}\left\|\frac{\partial \eta^{k}}{\partial t}(\cdot, s)\right\|_{L^{2}(\partial \Omega)} .
$$

In (64), $\left\|g^{\theta}(\cdot, s)\right\|_{L^{2}(\partial \Omega)}<+\infty$ since $g^{\theta} \in C^{0}\left([0, T] ; L^{2}(\Omega)\right)$, and $\left\|\eta^{k}(\cdot, s)\right\|_{L^{2}(\partial \Omega)}=1$ since $\eta^{k}$ are $L^{2}$ normalized POD modes. We finally use the second assumption to conclude: since $\frac{\partial g^{\theta}}{\partial t} \in$ $\left.C^{0}\left([0, T] ; L^{2}(\partial \Omega)\right), \forall \boldsymbol{\theta} \in\right] 0,1\left[{ }^{p}\right.$, we deduce that $\frac{\partial \eta^{k}}{\partial t} \in C^{0}\left([0, T] ; L^{2}(\partial \Omega)\right)$, by linearity of the POD modes with respect to the snapshots. This allows us to conclude the proof since

$$
\left\|\sum_{k=1}^{K_{B C}} \dot{c}_{k}^{\theta}(s) \xi^{k}(\cdot, s)\right\|_{L^{2}(\Omega)}<+\infty
$$

holds, $\forall s \in[0, T], \forall K_{B C}$.

\subsection{Overview of the proposed methodology}

Gathering all the previous steps of the ROM methodology, namely eqs. (4), (31), (37) for the first-level ROM, and (44) for the second-level ROM, the approximate solution $\widehat{u}^{\theta}$ can be written as

$$
\begin{aligned}
\widehat{u}^{\theta}(\mathbf{x}, t)= & \sum_{k=1}^{K_{I V}} a_{k}^{\boldsymbol{\theta}}(t) \varphi_{I V}^{k}(\mathbf{x})+\sum_{k=1}^{K_{B C}}\left(g^{\boldsymbol{\theta}}(\cdot, t), \eta^{k}(\cdot, t)\right)_{L^{2}(\partial \Omega)} \sum_{l=1}^{L^{k}} \sum_{m=1}^{M^{k}} \beta_{l m}^{k} \chi^{k, l}(\mathbf{x}) \varsigma^{k, m}(t) \\
& +\sum_{k=1}^{K} \sum_{m=1}^{M} \alpha_{k m}(\boldsymbol{\theta}) \varphi^{k}(\mathbf{x}) \xi^{m}(t) .
\end{aligned}
$$

The different steps of the proposed ROM methodology are summarized in Algorithms 1 and 2 , where $\boldsymbol{\theta}$ denotes any design parameter point. Algorithm 1 outlines the steps involved in computing the ROM solution of the full non-linear problem, $\widehat{u}^{\theta}$, given by (65). Algorithm 2 outlines the steps involved in computing the ROM solution of the auxiliary parabolic linear PDE, $\widehat{v}^{\theta}$, corresponding to the two first terms of (65). 

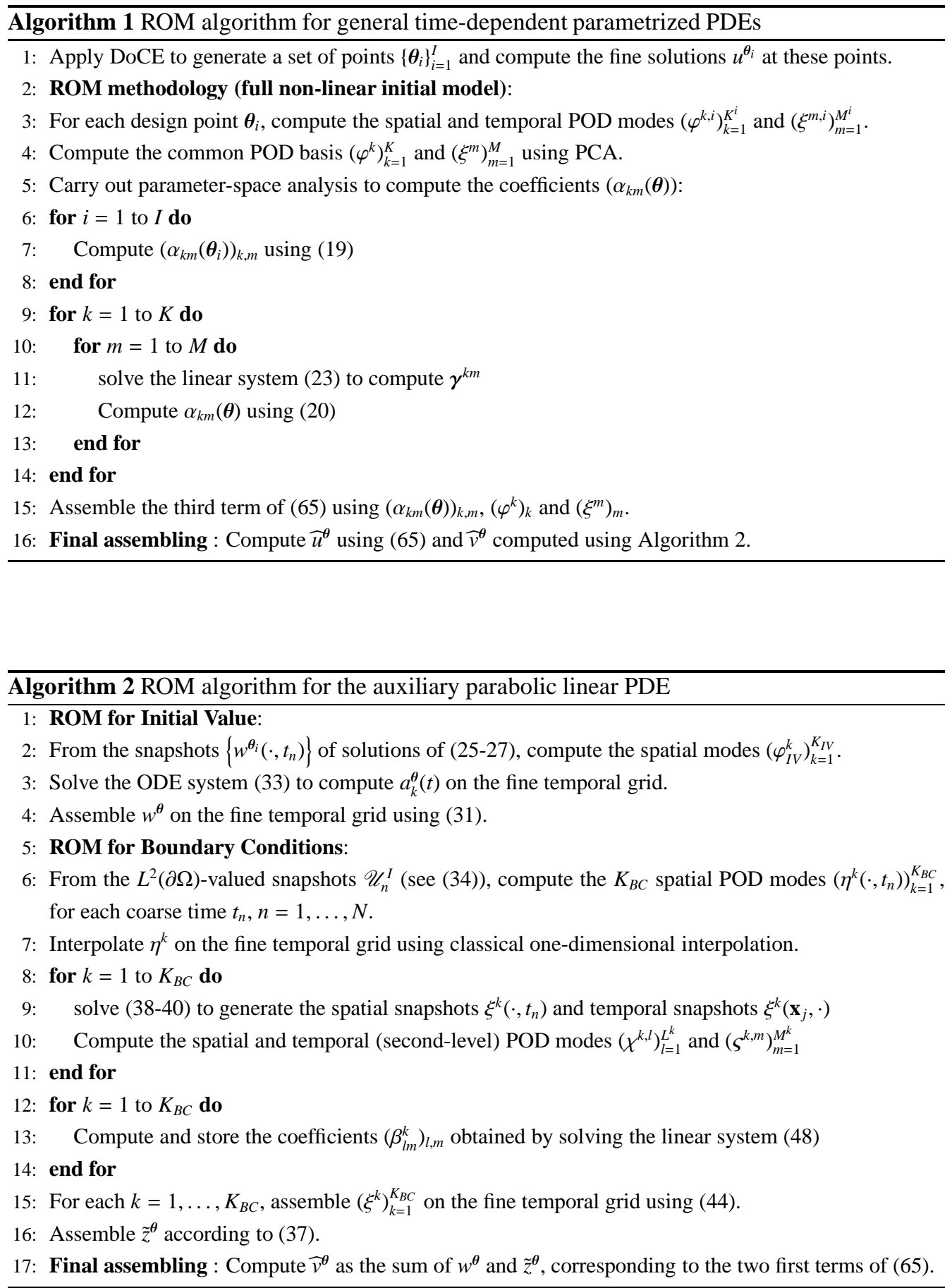


\section{Numerical test-case (1d-x $1 d-\theta$ 1d-t)}

\subsection{Definition of the test-case}

To illustrate the proposed methodology, we first consider the following unsteady Burgers' equation in $\Omega \times[0, T]$

$$
\left\{\begin{array}{c}
\frac{\partial u^{\theta}}{\partial t}+u^{\theta} \frac{\partial u^{\theta}}{\partial x}=0 \text { in } \Omega=[-2,2] \\
u^{\theta}(-2, t)=u_{L}(\theta), u^{\theta}(2, t)=u_{R}(\theta) \\
u_{\left.\right|_{t=0} ^{\theta}}^{\theta}=u_{0}^{\theta}
\end{array}\right.
$$

where the continuous initial data is given by

$$
u_{0}^{\theta}(x)=\left\{\begin{array}{lc}
u_{L}(\theta)=1+0.1 \theta & x \in[-2,-a], \\
-\frac{u_{L}(\theta)}{a} & x \in[-a, a], \\
u_{R}(\theta)=-u_{L}(\theta) & x \in[a, 2]
\end{array}\right.
$$

with $a \in] 0,2[$. The non-linear problem (66-67) is inspired from [26] where a randomly parametrized Burgers' equation with an initial shock is studied. The solution of (66-67) is continuous for time $t \in\left[0, a / u_{L}(\theta)[\right.$ and is given by

$$
u^{\theta}(x, t)= \begin{cases}u_{L}(\theta) & x<0 \\ u_{R}(\theta) & x>0\end{cases}
$$

for times $t \geq a / u_{L}(\theta)$, since $u^{\theta}$ is a shock solution travelling with the speed $s(\theta)=\frac{1}{2}\left(u_{L}(\theta)+\right.$ $\left.u_{R}(\theta)\right)=0$. In our numerical experiments, we will consider an integration time $T<a / \max _{\theta}\left|u_{L}(\theta)\right|$ in order to study the transient phase, namely before the shock appears.

\subsection{Numerical results}

Since the boundary conditions in (66) do not vary as a function of time, the ROM (4) boils down to

$$
\widehat{u}^{\theta}(x, t)=u_{0}^{\theta}(x)+\sum_{k=1}^{K} \sum_{m=1}^{M} \alpha_{k m}(\theta) \varphi^{k}(x) \xi^{m}(t)
$$

meaning that we don't need to solve an auxiliary PDE of the form (6-8). This simple test-case allows us to validate the first level of our ROM methodology described in Sections 3 and 4.

In our numerical experiments, we take $a=1, T=0.8 \mathrm{~s}$ and $\theta \in[-2,2]$. We consider a set of $I=20$ design points $\theta_{i}$ uniformly spread in $[-2,2]$ for the parameter-space sampling and a 
uniform spatial grid made of $N_{x}=100$ points. For the coarse temporal sampling, we use $N=51$ points in $[0, T]$ to generate the spatial snapshots. We consider a coarse spatial mesh of $J=50$ points $x_{j}$ and a time-step $\delta t=4.25 \times 10^{-3}$ to generate the temporal snapshots, corresponding to a grid of $N_{t}=201$ points.

In order to compute the spatial and temporal basis functions $\varphi^{k}$ and $\xi^{m}$ needed in the expansion (69), we first compute, for each design parameter $\boldsymbol{\theta}_{i} \in \mathscr{W}^{I}, K^{i}$ spatial modes $\varphi^{k, i}$ and $M^{i}$ temporal modes $\xi^{m, i}$, by applying PCA methods. Using thresholds of $\epsilon=10^{-3}$, we get a total of $\sum_{i=1}^{I} K^{i}=105$ and $\sum_{i=1}^{I} M^{i}=105$ POD modes. By applying another PCA method on these different sets of modes with thresholds of $\epsilon=10^{-2}$, we finally retain a set of common POD modes $\left(\varphi^{k}\right)_{k=1, \ldots, K}$ and $\left(\xi^{m}\right)_{m=1, \ldots, M}$, with $K=8$ and $M=7$.

For the estimation of the coefficients $\alpha_{k m}(\theta)$, we use a Gaussian radial basis function $\Phi(r)=$ $e^{-\frac{t^{2}}{2 \sigma}}$ with $\sigma=0.13$ in (20). This optimal value for $\sigma$ has been numerically obtained by minimizing the distance between $\alpha_{k m}\left(\theta_{i}\right)$ given by (19) and their RBF approximations (20).

As a first illustration, we compare on Figure 1 the approximate ROM solution $\widehat{u}^{\theta}$ to the exact solution $u^{\theta}$ for $\theta=2.0$, which is the maximal value of the parameter space interval. One can see a good agreement between the two solutions, even if small oscillations appears in the ROM approximation at the final time $T$.

For a more systematic comparison, we plot on Figure 2 different $L^{2}$ errors between the exact and the ROM solutions when the parameter $\theta$ belongs to a fine grid made of 200 points uniformly spread in $[-2,2]$. For each design parameter, we represent the maximal $L^{2}$ error

$$
\max _{t_{n} \in[0, T]}\left\|\widehat{u}^{\theta}\left(\cdot, t_{n}\right)-u^{\theta}\left(\cdot, t_{n}\right)\right\|_{L^{2}(\Omega)}
$$

and the relative $L^{2}$ error

$$
\frac{1}{N_{t}} \sum_{n=1}^{N_{t}} \frac{\left\|\widetilde{u}^{\theta}\left(\cdot, t_{n}\right)-u^{\theta}\left(\cdot, t_{n}\right)\right\|_{L^{2}(\Omega)}}{\left\|u^{\theta}\left(\cdot, t_{n}\right)\right\|_{L^{2}(\Omega)}}
$$

showing a good level of accuracy of the ROM (69).

\section{Numerical test-case (2d-x 3d- $\theta$ 1d-t)}

\subsection{Definition of the test-case}

To illustrate the whole ROM approach described in Sections 3, 4 and 5, we consider a more complex parametrized model test-case, where the parameters are involved within the initial value, the boundary conditions and the governing equation of the model. In this case, we will consider the ROM in its more advanced form (65), where we approximate the split solution (24) of the auxiliary PDE problem using the second-level ROM (44). 

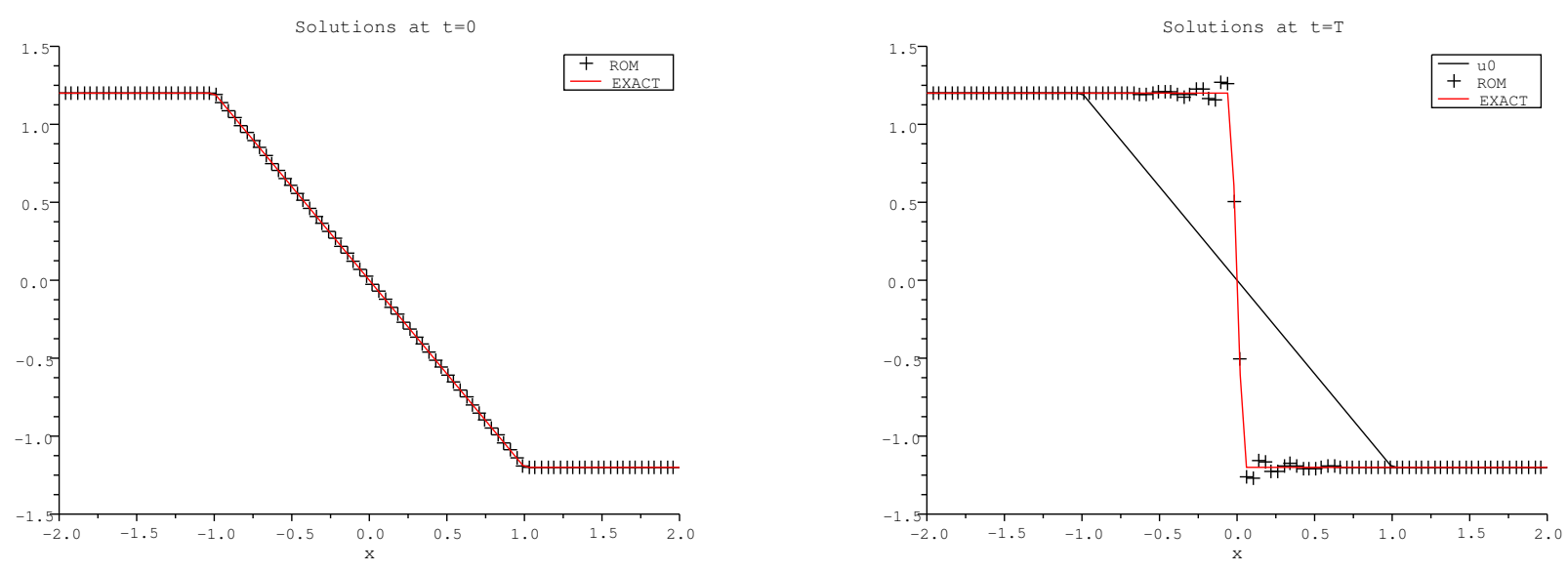

$$
u^{\theta}(\cdot, t) \text { and } \widehat{u}^{\theta}(\cdot, t) \text { at } t=0
$$

$u^{\theta}(\cdot, t)$ and $\widehat{u}^{\theta}(\cdot, t)$ at $t=T$
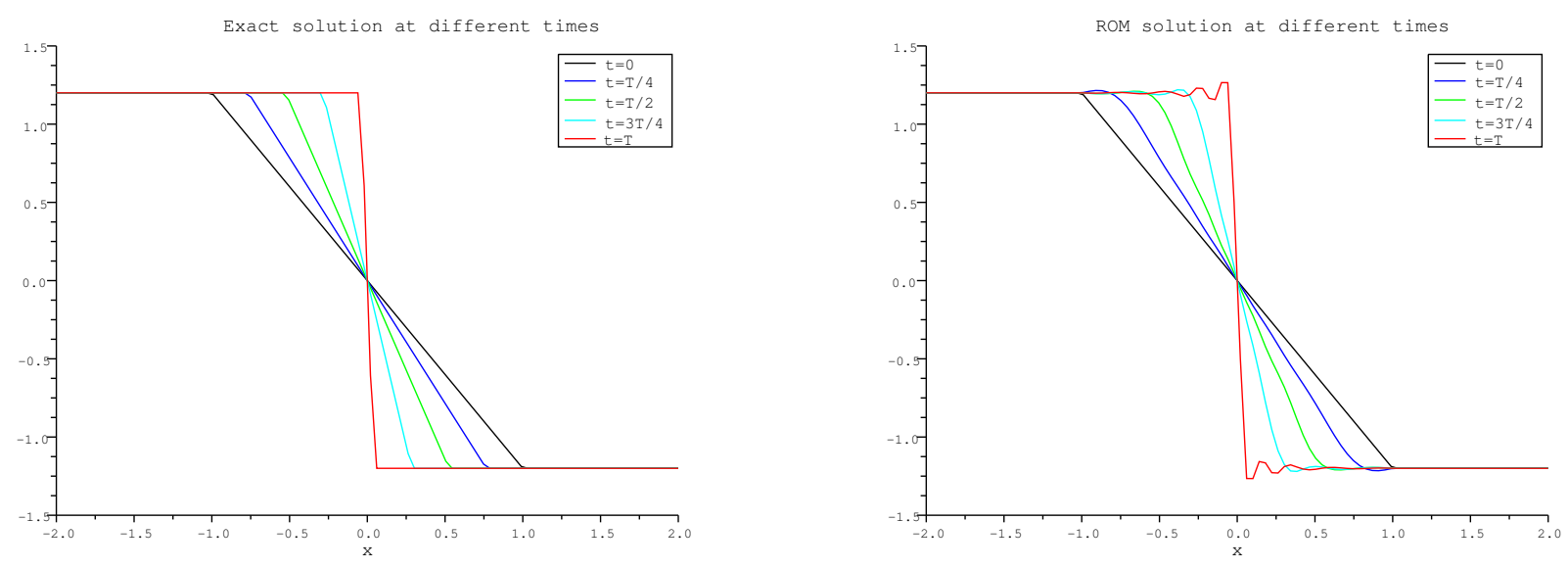

exact snapshots $u^{\theta}\left(\cdot, t_{n}\right)$

ROM snapshots $\widehat{u}^{\theta}\left(\cdot, t_{n}\right)$

Figure 1: Comparison of the exact and ROM solutions for $\theta=2.0$.

\subsubsection{Parametrization of the equations}

To illustrate the application of the proposed methodology, we consider the following unsteady convection-reaction-diffusion problem in $\Omega \times[0, T]$

$$
\left\{\begin{array}{l}
\left.\frac{\partial u}{\partial t}+\nabla \cdot\left(\tau \frac{u^{2}}{2}\right)-v \Delta u=f^{\theta_{1}} \text { in } \Omega=\right] 0,1\left[^{2},\right. \\
u=g^{\theta_{2}} \text { on } \Gamma \text { (upper BC), } \\
u=0 \text { on } \partial \Omega \backslash \Gamma, \\
u_{\left.\right|_{t=0}}=u_{0}^{\theta_{3}}
\end{array}\right.
$$




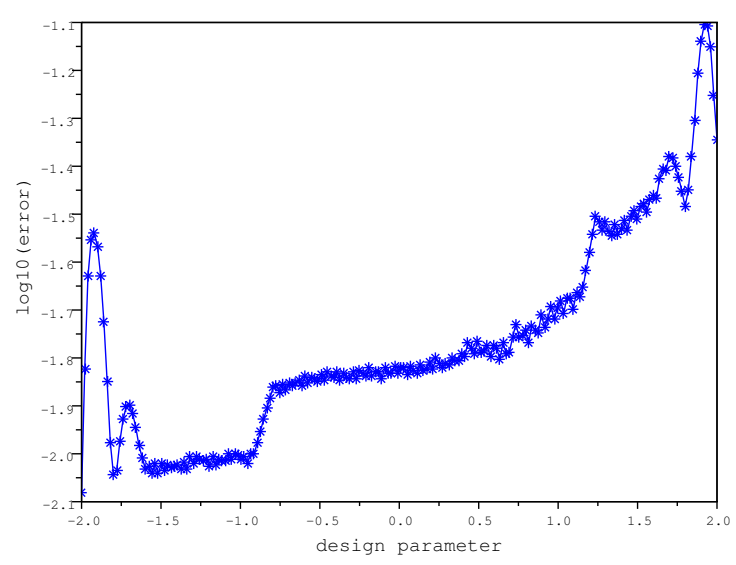

maximal $L^{2}$ errors (70)

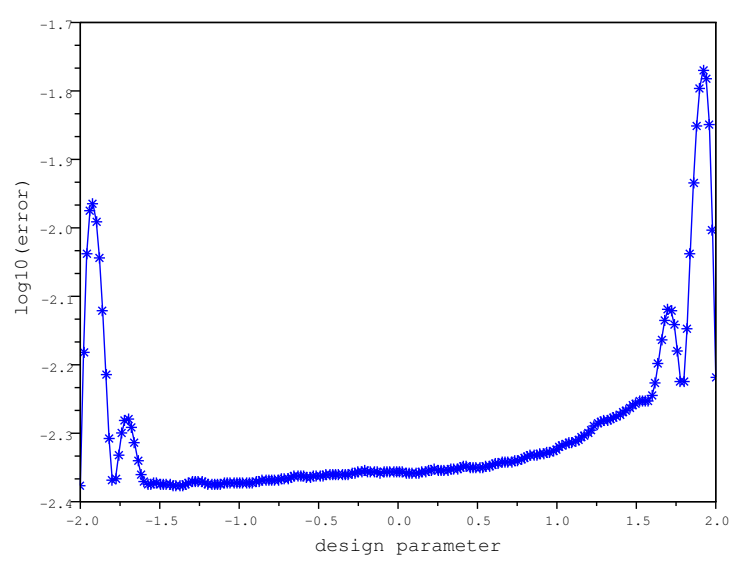

relative $L^{2}$ errors (71)

Figure 2: $L^{2}$ errors in $\log _{10}$ scale between the ROM and the exact solution for $\theta$ defined on a fine parameter space grid.

with 3 parameters: one in the reactive term $\left(\theta_{1}\right)$, one in the $\mathrm{BC}\left(\theta_{2}\right)$ and one in the initial condition $\left(\theta_{3}\right)$. Here $v$ denotes the viscosity, $\tau(x, y)$ is a rotating field defined by

$$
\tau(x, y)=\frac{1}{\|(-(y-1 / 2), x-1 / 2)\|}\left(\begin{array}{c}
-(y-1 / 2) \\
x-1 / 2
\end{array}\right)
$$

and the reactive term is given by

$$
f^{\theta_{1}}=0.1-0.01 \theta_{1} u|u|
$$

This model is an extension of the steady-state parametrized PDE model studied in [10].

\subsubsection{Parametrization of the $B C$}

We justify here the chosen form of the parametrized BC, since there exists many possible choices for the function $g^{\theta}$. First of all $g^{\theta}$ must satisfy the condition $g^{\theta}(\cdot, 0)=0$, because of eqs. (29-30). Moreover, considering an usual expression for $g^{\theta}$, namely as the product of a temporal function and a spatial one, is restrictive. It is worth noting that if $g^{\theta}(\mathbf{x}, t)=\alpha(t) \beta^{\theta}(\mathbf{x})$, then it can be seen from (34) that for different times $t_{n} \neq t_{m}$, the snapshots of $\mathscr{U}_{n}^{I}$ and $\mathscr{U}_{m}^{I}$ are colinear since

$$
g^{\theta_{i}}\left(\cdot, t_{n}\right)=\frac{\alpha\left(t_{n}\right)}{\alpha\left(t_{m}\right)} g^{\theta_{i}}\left(\cdot, t_{m}\right), \forall i=1, \ldots I .
$$

Therefore we get the same eigenfunctions $\eta_{B C}^{k}$ by performing a PCA on the sets $\mathscr{U}_{n}^{I}$ or $\mathscr{U}_{m}^{I}$. In order to consider the most general case, namely time-dependent POD modes $\eta_{B C}^{k}$, we use the 
following expression for $g^{\theta}$

$$
g^{\theta}(\mathbf{x}, t)=\alpha_{1}(t) \beta_{1}^{\theta}(\mathbf{x})+\alpha_{2}^{\theta}(t)
$$

with $\alpha_{1}(0)=\alpha_{2}^{\theta}(0)=0$ since $g^{\theta}(\cdot, 0)=0$.

In our numerical simulations we take

$$
\begin{gathered}
\alpha_{1}(t)=\sin \left(\frac{\pi t}{2 T}\right), \\
\beta_{1}^{\theta_{2}}(x)=1-\theta_{2} \sin \left(2 \pi\left(x-\frac{1}{2}\right)\right),
\end{gathered}
$$

and

$$
\alpha_{2}^{\theta_{2}}(t)=\frac{\theta_{2} t}{T} .
$$

\subsubsection{Parametrization of the initial value}

For parametrization of $u_{0}$, we consider some perturbations of a known function $\bar{u}$ in the form

$$
u_{0}^{\theta}(\mathbf{x})=\bar{u}(\mathbf{x})+\boldsymbol{\theta} c(\mathbf{x})
$$

with $\bar{u}_{\partial \Omega \Omega}=c_{l a \Omega}=0$, because of the homogeneous BC of the heat problem (25-27). The relation (79) can be viewed as a simplified representation of Karhunen-Loève expansions used for modeling random fields in a stochastic framework (see [22]), where $\bar{u}$ is a deterministic mean function and $\boldsymbol{\theta}$ are random variables.

In our numerical simulations $\boldsymbol{\theta}=\theta_{3}$ and $\bar{u}$ is taken as an harmonic function that satisfies the Poisson problem

$$
\left\{\begin{array}{l}
-\Delta \bar{u}=f \text { in } \Omega, \\
\bar{u}=0 \text { on } \partial \Omega,
\end{array}\right.
$$

with $f \equiv 1$. We choose

$$
c(\mathbf{x})=\lambda\left(x+\frac{1}{2}\right)\left(x-\frac{1}{2}\right)\left(y+\frac{1}{2}\right)\left(y-\frac{1}{2}\right),
$$

with $\lambda=\frac{8}{5} \max _{\Omega}|\bar{u}|$ so that the maximal amplitude of $c$ represent a tenth of $\max _{\Omega}|\bar{u}|$.

\subsection{Numerical results: approximation of the auxiliary PDE}

In order to validate the methodology presented in Section 5, we first present numerical results for approximation of the auxiliary PDE model (6-8). The BC conditions and the initial value are defined by (75-78) and (79-81), respectively, meaning that we consider a two-parameter problem. 


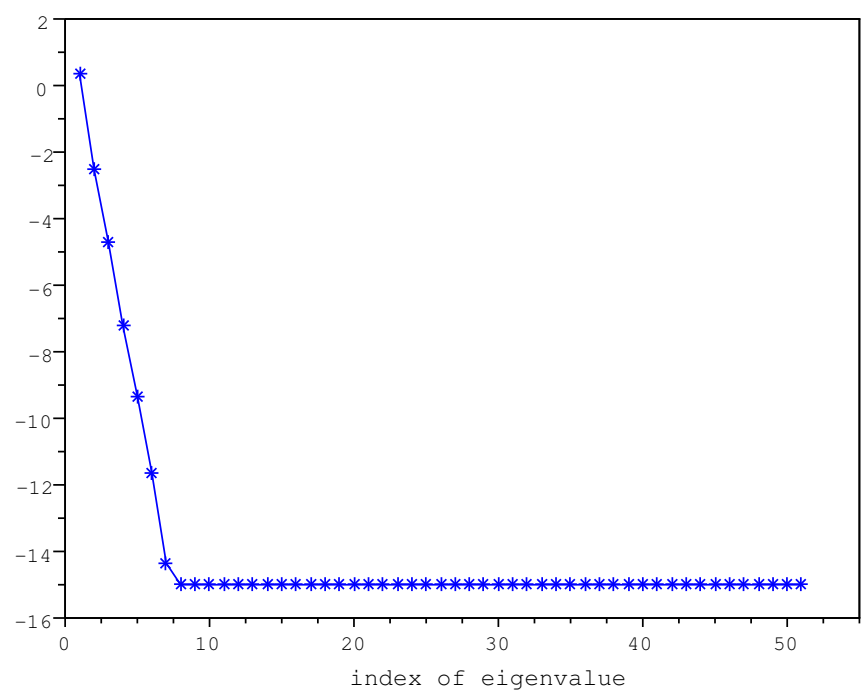

Figure 3: Computation of $\varphi_{I V}^{k}$ : spectrum of the Gram matrix in $\log _{10}$ scale. We retain $K_{I V}=6$ spatial modes.

After presenting the different parameters chosen for these simulations, we will compare the ROM solution

$$
\begin{aligned}
\widehat{v}^{\theta_{2}, \theta_{3}}(\mathbf{x}, t) & =\sum_{k=1}^{K_{I V}} a_{k}^{\theta_{3}}(t) \varphi_{I V}^{k}(\mathbf{x}) \\
& +\sum_{k=1}^{K_{B C}}\left(g^{\theta_{2}}(\cdot, t), \eta^{k}(\cdot, t)\right)_{L^{2}(\partial \Omega)} \sum_{l=1}^{L^{k}} \sum_{m=1}^{M^{k}} \beta_{l m}^{k} \chi^{k, l}(\mathbf{x}) \varsigma^{k, m}(t),
\end{aligned}
$$

which corresponds to the two first terms of (65), to the solution $v^{\theta_{2}, \theta_{3}}$ obtained by directly solving (6-8) with a precise implicit Euler scheme.

We choose the following parameters for our simulations: $T=2 \mathrm{~s}, N_{x}=20$ points per spatial direction, and a time-step $\delta t=0.002$ for the fine Euler resolution. For the heat problem resolution with homogeneous BC (see $\S 5.2$ ), we take $N=51$ points for the temporal sampling, half of them being uniformly spread in $[0,0.2]$ to capture the unsteady behavior of the solution $w^{\theta_{3}}$. Applying PCA with a threshold of $\varepsilon=10^{-12}$ leads to $K_{I V}=6$ spatial modes $\varphi_{I V}^{k}$ (see Figure 3 ). Figure 4 shows the time history of each coefficient $a_{k}^{\theta_{3}}(t)$ of the expansion (31). It can be seen that each coefficient converges towards 0 , which is consistent with the fact that we analyze the solution with respect to its initial value dependency.

Concerning the heat problem with null initial value (see $\S 5.3$ ), we sample the parameter $\theta_{2}$ 

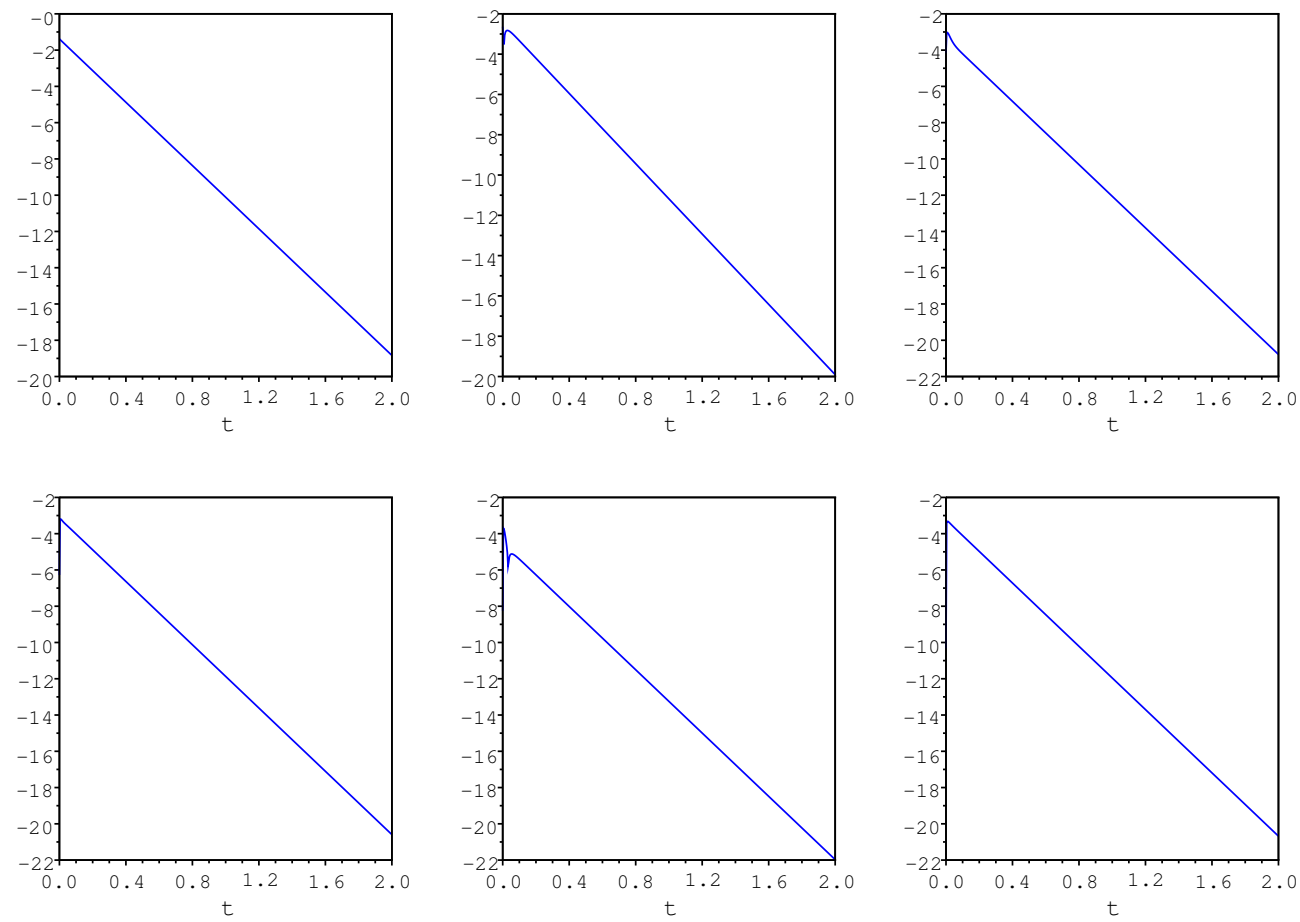

Figure 4: Time history of the coefficients $a_{k}^{\theta_{3}}(t), k=1, \ldots, K_{I V}$ in $\log _{10}$ scale. It can be seen that all the coefficients vanish for large times.

with $I=7$ values uniformly spread in $[0,1]$. Applying PCA with a threshold of $\varepsilon=10^{-6}$ leads to $K_{B C}=2$ spatial modes $\eta^{k}$ (see Figure 5(a)).

For the second-level ROM (44) we store, for each $k=1, \ldots, K_{B C}, N_{T}=N$ spatial snapshots $\left(\xi^{k}\left(\cdot, t_{n}\right)\right)_{n}$ and $N_{X}=200$ temporal series $\left(\xi^{k}\left(\mathbf{x}_{j}, \cdot\right)\right)_{j}$ where $\mathbf{x}_{j}$ belongs to a coarse spatial grid uniformly spread in $\Omega$. Applying PCA methods with thresholds of $\varepsilon=10^{-9}$ in both cases leads to $L^{k}=7$ spatial modes $\chi^{k, l}(\mathbf{x})$ and $M^{k}=8$ temporal modes $\varsigma^{k, m}(t)$, for each $k$ (see Figures $5(\mathrm{~b})$ and $5(\mathrm{c}))$.

For $\theta_{2}=0.2$ and $\theta_{3}=0.1$ we now compare the approximate ROM solution to the fine Euler solution. The results presented in Figures 6 and 7 show a good agreement between the approximated and fine Euler solutions. The mean $L^{2}$ relative error is

$$
\frac{1}{N} \sum_{n=1}^{N} \frac{\left\|\widehat{v}^{\theta_{2}, \theta_{3}}\left(\cdot, t_{n}\right)-v^{\theta_{2}, \theta_{3}}\left(\cdot, t_{n}\right)\right\|_{L^{2}(\Omega)}}{\left\|v^{\theta_{2}, \theta_{3}}\left(\cdot, t_{n}\right)\right\|_{L^{2}(\Omega)}} \simeq 7.47 \times 10^{-2}
$$




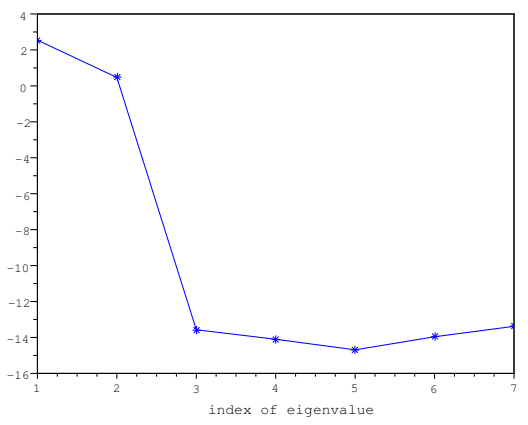

(a)

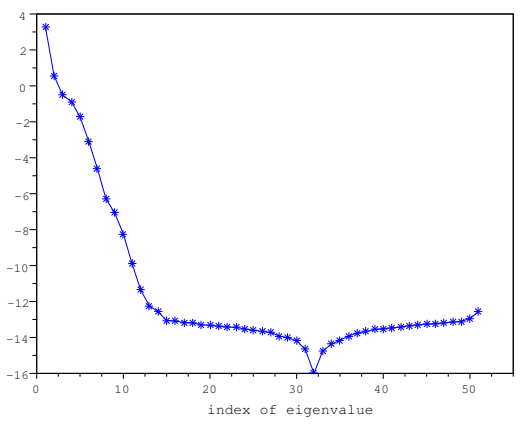

(b)

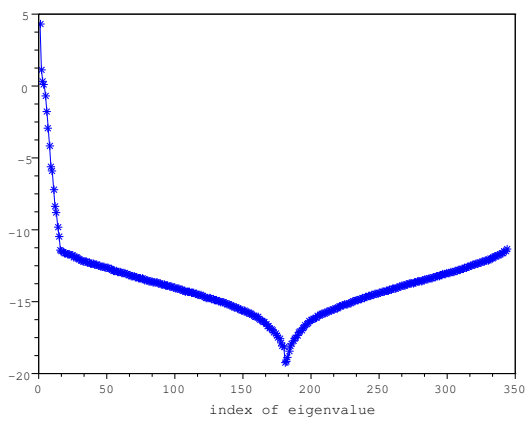

(c)

Figure 5: (a) - Computation of $\eta^{k}$ : spectrum of the Gram matrix in $\log _{10}$ scale. We retain $K_{B C}=2$ spatial modes. (b) Computation of $\chi^{k, l}$ : spectrum of the Gram matrix in $\log _{10}$ scale. We retain $L^{k}=7$ spatial modes. (c) - Computation of $\varsigma^{k, m}$ : spectrum of the Gram matrix in $\log _{10}$ scale. We retain $M^{k}=8$ temporal modes.

It is worth noting that on the upper $\mathrm{BC} \Gamma$, the solution is better approximated:

$$
\frac{1}{N} \sum_{n=1}^{N} \frac{\left\|\widehat{v}^{\theta_{2}, \theta_{3}}\left(\cdot, t_{n}\right)-v^{\theta_{2}, \theta_{3}}\left(\cdot, t_{n}\right)\right\|_{L^{2}(\Gamma)}}{\left\|v^{\theta_{2}, \theta_{3}}\left(\cdot, t_{n}\right)\right\|_{L^{2}(\Gamma)}} \simeq 4.99 \times 10^{-2} .
$$

The $L^{2}$ relative errors over the whole domain $\Omega$ and the upper boundary $\Gamma$ as a function of time is shown in Figures 8(a) and 8(b), respectively. It can be noted that the quality of the approximated solution is not affected by the size of the temporal coarse grid used for the modes $\eta^{k}$. We maintain the same level of accuracy using a temporal undersampling, with a coarse grid made of only 21 points. Figures 9(a) and 9(b) compare the temporal evolution of the solution at a fixed point in the interior and boundary for the ROM and the direct Euler solution. 

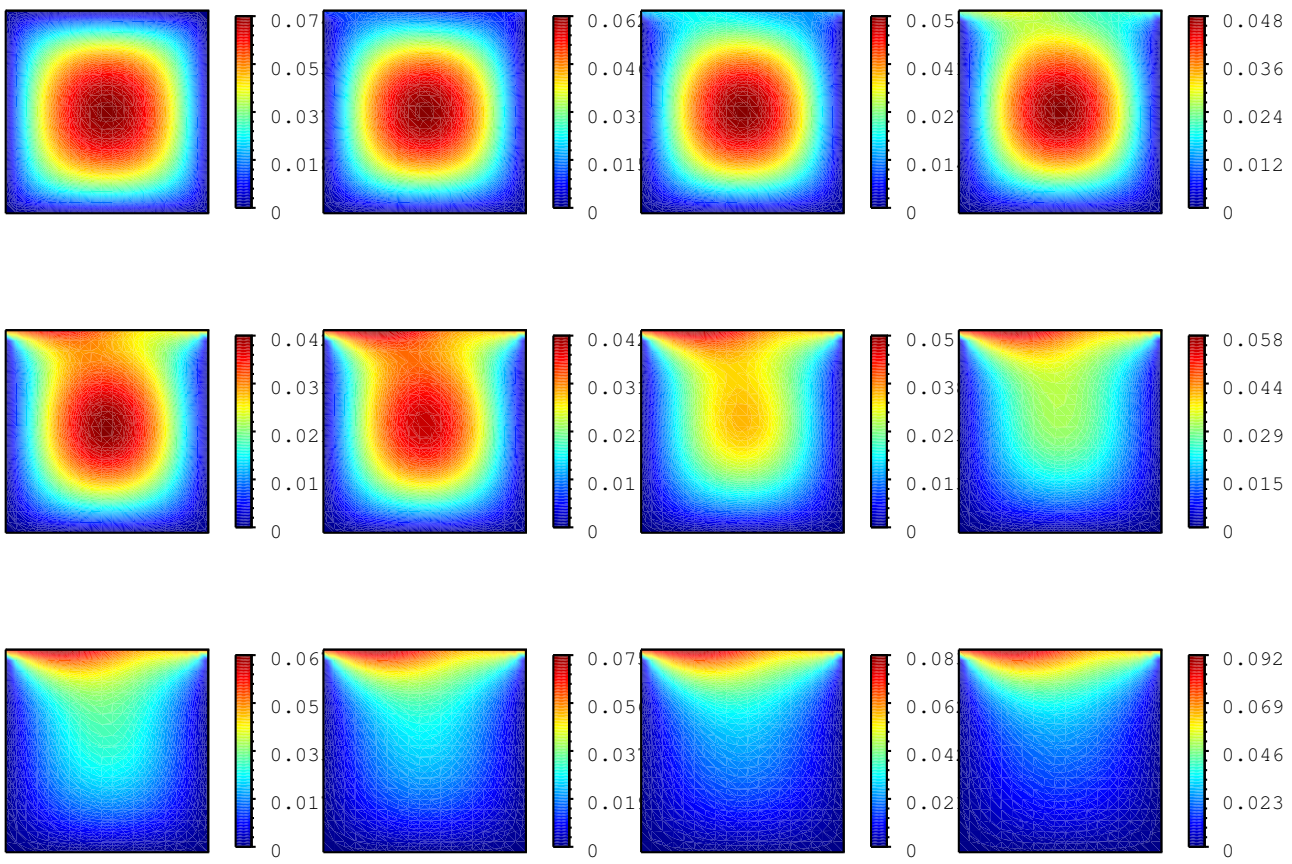

Figure 6: First few snapshots of the direct implicit Euler solution $v^{\theta_{2}, \theta_{3}}\left(\cdot, t_{n}\right), n=1, \ldots, 12$, for $\theta_{2}=0.2$ and $\theta_{3}=0.1$.

We would like to point out that computation and storage of the POD modes $\eta^{k}\left(\cdot, t_{n}\right)$ on the coarse temporal grid require a numerical post-processing. Since these modes are obtained for different times $t_{n}$, it is possible to generate discontinuities, because eigenvectors in the PCA methods are defined up to the sign. A simple procedure is hence needed to detect the possible changes of sign and has been used in our simulations.

\subsection{Numerical results: full non-linear problem}

We present now numerical results corresponding to the full non-linear problem (72), with the parametrization described in Sections 7.1.1, 7.1.2 and 7.1.3. For our numerical simulations we take $v=0.05$ and $T=5 \mathrm{~s}$. The fine spatial grid is made of $20 \times 20$ points and the coarse one of $J=$ 344 points uniformly spread in $\Omega$, including the upper BC. For the temporal discretization, we take $N=101$ points to generate the spatial snapshots (12) and $N_{\text {fine }}=501$ for the snapshots time series (14), half of them being uniformly spread in $[0,0.5]$. For the parameter-space discretization we use a regular grid of $I=5^{3}=125$ design points. 

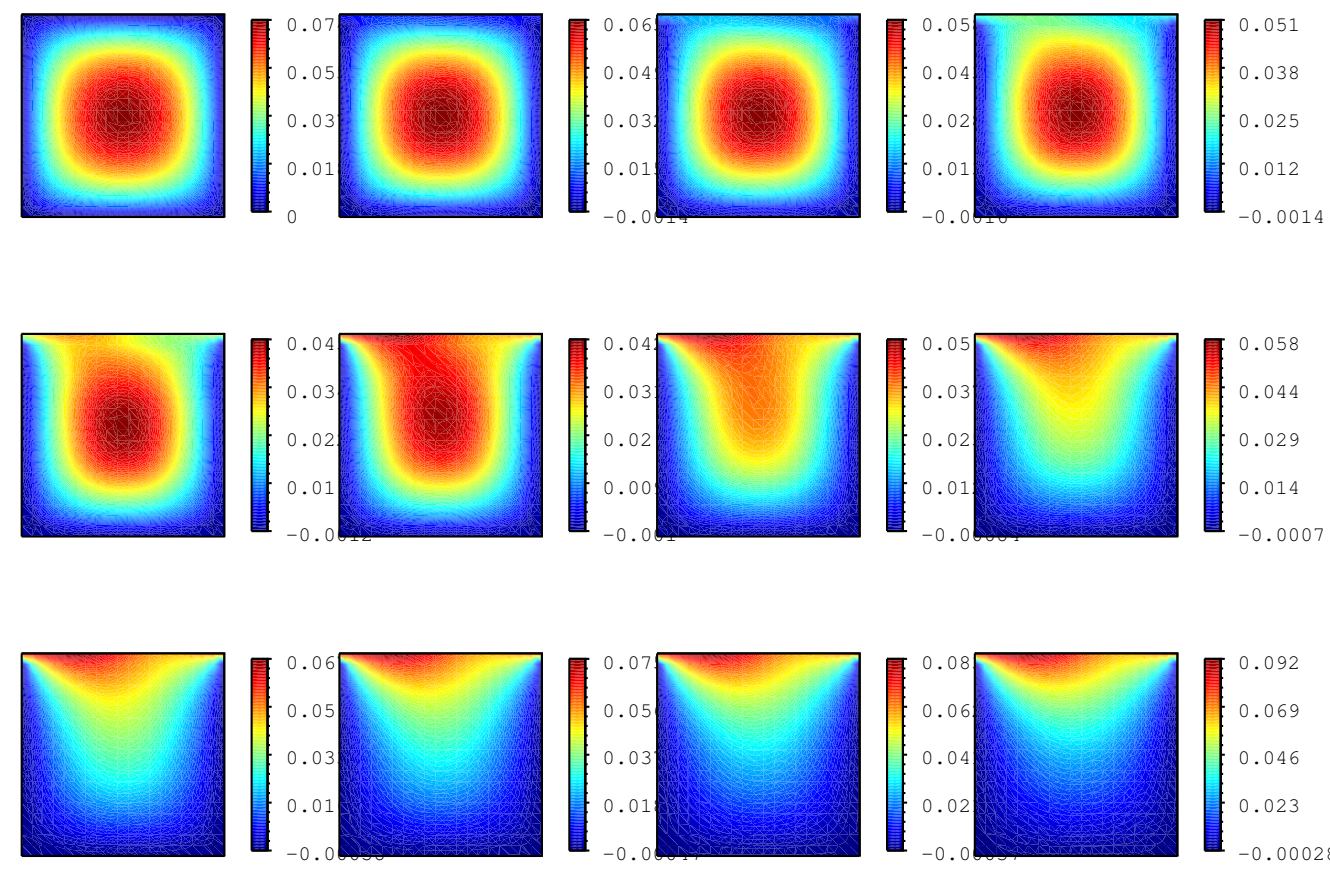

Figure 7: First few snapshots of the ROM solution $\widehat{v}^{\theta_{2}, \theta_{3}}\left(\cdot, t_{n}\right), n=1, \ldots, 12$ given by eq. (82), for $\theta_{2}=0.2$ and $\theta_{3}=0.1$.

We proceed as follows to construct the spatial and temporal basis functions, $\varphi^{k}$ and $\xi^{m}$, needed in the expansion (4). For each design parameter $\boldsymbol{\theta}_{i} \in \mathscr{W}^{I}, K^{i}$ spatial modes $\varphi^{k, i}$ and $M^{i}$ temporal modes $\xi^{m, i}$ are computed by applying PCA methods. Using thresholds respectively of $\epsilon=10^{-4}$ and $\epsilon=10^{-5}$, we get a total of $\sum_{i=1}^{I} K^{i}=500$ and $\sum_{i=1}^{I} M^{i}=375$ POD modes. From these different sets of POD modes linked to each design parameter, we deduce common spatial and temporal basis sets applying PCA once again on the families

$$
\left\{\left(\varphi^{k, i}\right)_{k=1, \ldots, K^{i}}\right\}_{i=1, \ldots, I}
$$

and

$$
\left\{\left(\xi^{m, i}\right)_{k=1, \ldots, M^{i}}\right\}_{i=1, \ldots, I} .
$$

Considering thresholds of $\epsilon=10^{-5}$ in both cases, we obtain a set of common POD modes $\left(\varphi^{k}\right)_{k=1, \ldots, K}$ and $\left(\xi^{m}\right)_{m=1, \ldots, M}$, with $K=9$ and $M=4$. The corresponding eigenvalues of the Gram matrices are shown in Figure 10, showing a quick decay of the spectra. The spatial and temporal modes are depicted in Figures 11 and 12. It can be seen from Figure 12 that the conditions $\xi^{m}(0)=0$ are satisfied by the temporal basis functions. 


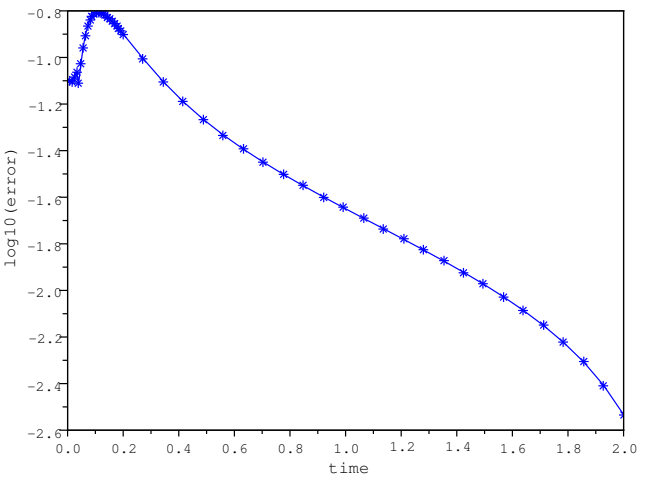

(a)

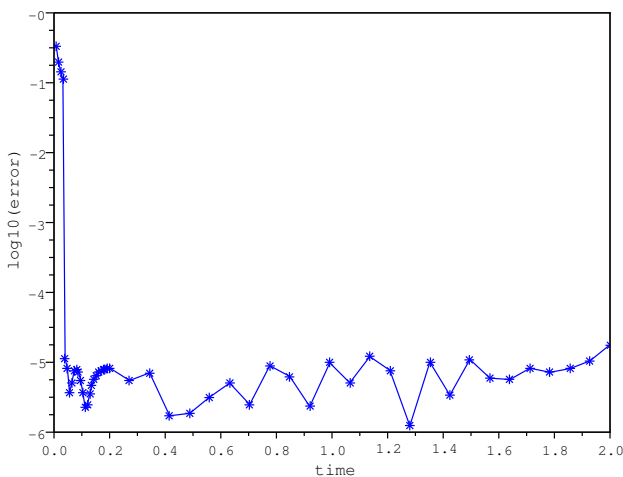

(b)

Figure 8: (a) $-L^{2}$ relative errors on the whole domain $\Omega$ between direct and ROM solutions, for each times $t_{n}$. (b) $-L^{2}$ relative errors on the upper boundary $\Gamma$ in $\log _{10}$ scale between direct and ROM solutions, for each times $t_{n}$.

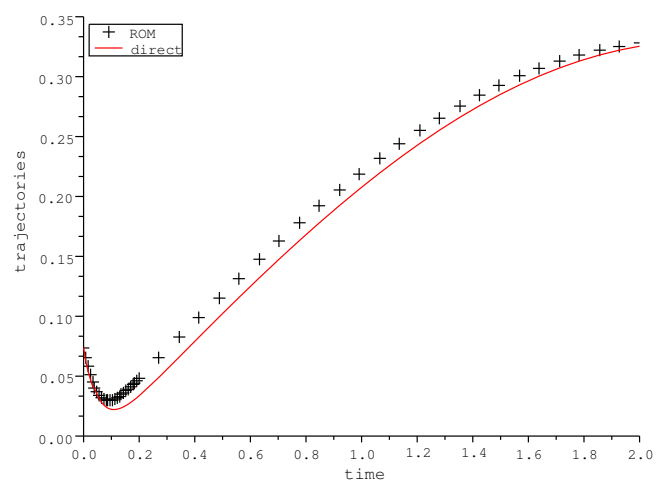

(a)

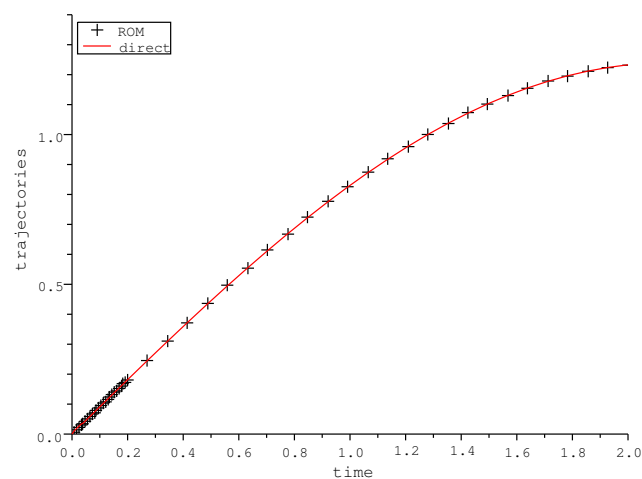

(b)

Figure 9: (a) - For a fixed point in the interior of $\Omega$, trajectory of the ROM and direct solutions. (b) - For a fixed point on the upper BC, trajectory of the ROM and direct solutions. 
In our simulations we using the Gaussian radial basis function $\Phi(r)=e^{-\frac{r^{2}}{2 \sigma}}$ with $\sigma=0.15$. This optimal value of $\sigma$ is numerically obtained by minimizing the maximal distance between the known coefficients $\alpha_{k m}\left(\boldsymbol{\theta}_{i}\right)$ given by (19), and their RBF estimations given by (20). Such a value of $\sigma$ leads to a maximal difference of $1.57 \times 10^{-4}$. The condition number of the interpolation matrix involved in (22) is around 15.8 for the chosen value of the scaling parameter $\sigma$.

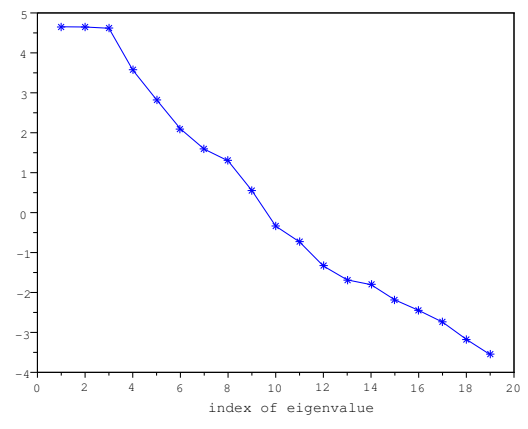

(a)

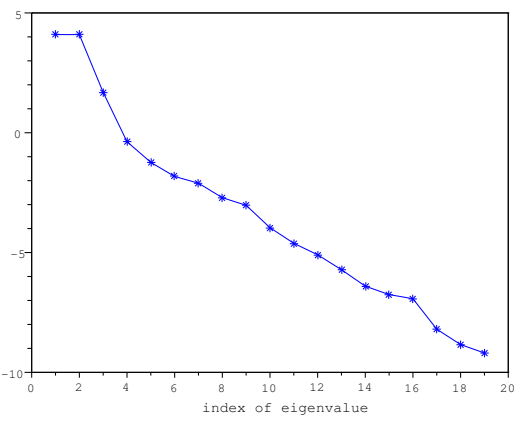

(b)

Figure 10: (a) - Computation of $\varphi^{k}$ : spectrum of the Gram matrix in $\log _{10}$ scale. (b) - Computation of $\xi^{m}$ : spectrum of the Gram matrix in $\log _{10}$ scale.

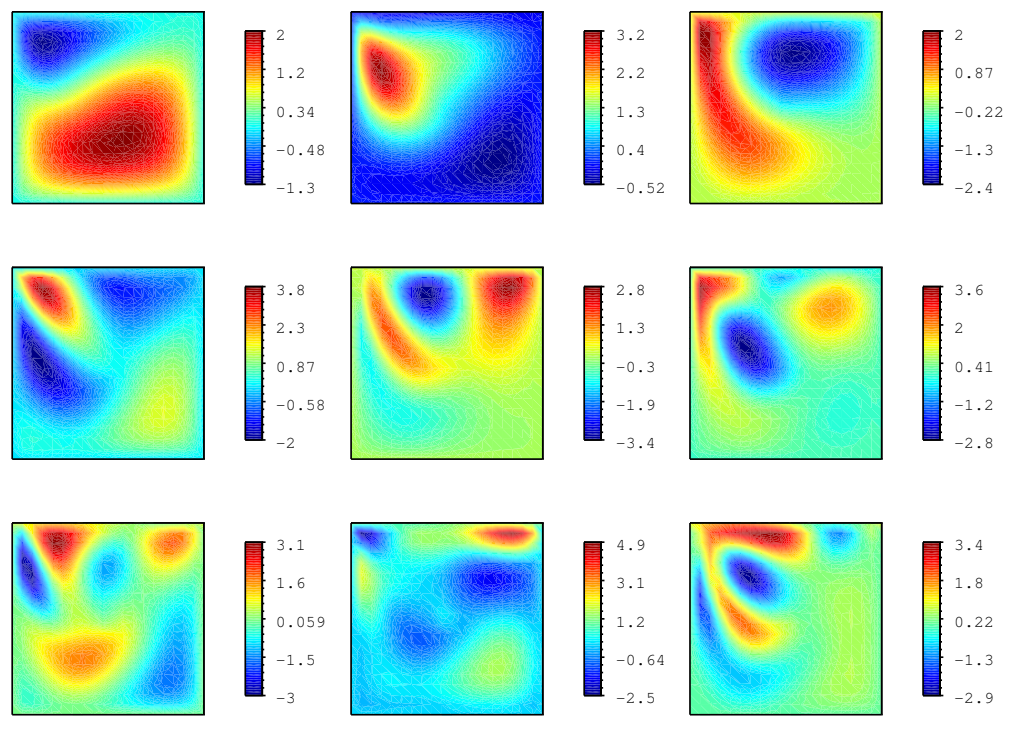

Figure 11: First 9 spatial basis functions $\varphi^{k}$. 

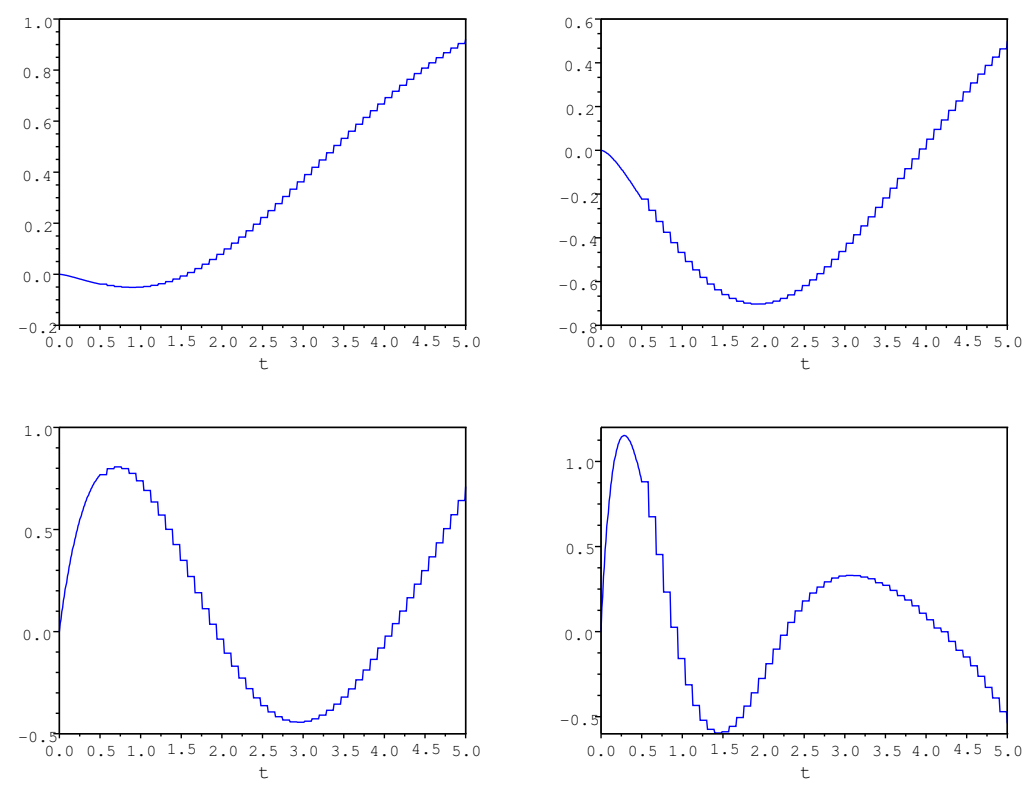

Figure 12: First 4 temporal basis functions $\xi^{m}$, with $\xi^{m}(0)=0$.

Concerning the initial value part of the ROM, we consider the same temporal sampling in $[0, T]$ used for the non-linear snapshots generation, to get the snapshots of the heat problem with null BC $(N=101)$. This leads us to consider $K_{I V}=4 \operatorname{modes} \varphi_{I V}^{k}$. For the BC part of the ROM, we consider 20 values of $\theta_{2}$ uniformly spread in $[0,1]$ in order to perform PCA on the set (34). This leads us to retain $K_{B C}=2$ POD modes $\xi^{k}$. The spatio-temporal modes $\xi^{k}$ are not computed exactly but are approximated by (44), with $M^{k}=L^{k}=5$, for each $k=1, \ldots, K_{B C}$.

Figures 13 and 14 show some temporal snapshots of the solution directly obtained by an implicit Euler scheme at the beginning and at the end of the simulation. We consider the parameter vector $\boldsymbol{\theta}_{0}=(0.27,0.92,0.23)^{T}$, which is not included in our snapshot generation process (12) and (14). The temporal snapshots approximated by the ROM for the same parameter $\boldsymbol{\theta}_{0}$ are shown in Figures 15 and 16. It can be seen that the reduced-order model provides a very accurate approximation of the full direct solution during all time-instants in the interval [0,5]. It is to be noticed that the snapshots directly generated by a fine Euler scheme are obtained with $N_{x}^{2} \times N_{T}=40400$ unknowns, while the ROM snapshots require $K_{I V}+\sum_{k=1}^{K_{B C}} L^{k} M^{k}+K M=90$ unknowns, once all the POD basis are generated. In addition, we represent in Figure 17 the normalized $L^{2}$ errors

$$
\mathcal{E}\left(t_{n}\right)=\frac{\left\|\widetilde{u}^{\theta_{0}}\left(\cdot, t_{n}\right)-u^{\theta_{0}}\left(\cdot, t_{n}\right)\right\|_{L^{2}(\Omega)}}{\max _{n}\left\|u^{\theta_{0}}\left(\cdot, t_{n}\right)\right\|_{L^{2}(\Omega)}}
$$

showing the ability of the ROM to reproduce accurately the full direct solution as a function of 
time. As another illustration, we give in Figures 18 and 19 a three-dimensional representation of these two solutions at initial and final time-instants, showing the good agreement between each other.

To illustrate the accuracy of the ROM at other points in the parameter space, we use the mean $L^{2}$ error defined below

$$
E(\boldsymbol{\theta})=\frac{1}{N} \sum_{n=1}^{N} \mathcal{E}\left(t_{n}\right),
$$

where $\mathcal{E}\left(t_{n}\right)$ is the normalized $L^{2}$ error at time instant $t_{n}$ defined earlier in (83). Figure 20 shows the mean $L^{2}$ errors (84) represented for the 125 design points of the DoCE. It can be seen from these results that the mean value of these errors is about $9 \times 10^{-3}$ with a maximum error equal to 0.0123 , showing that the ROM provides good accuracy.
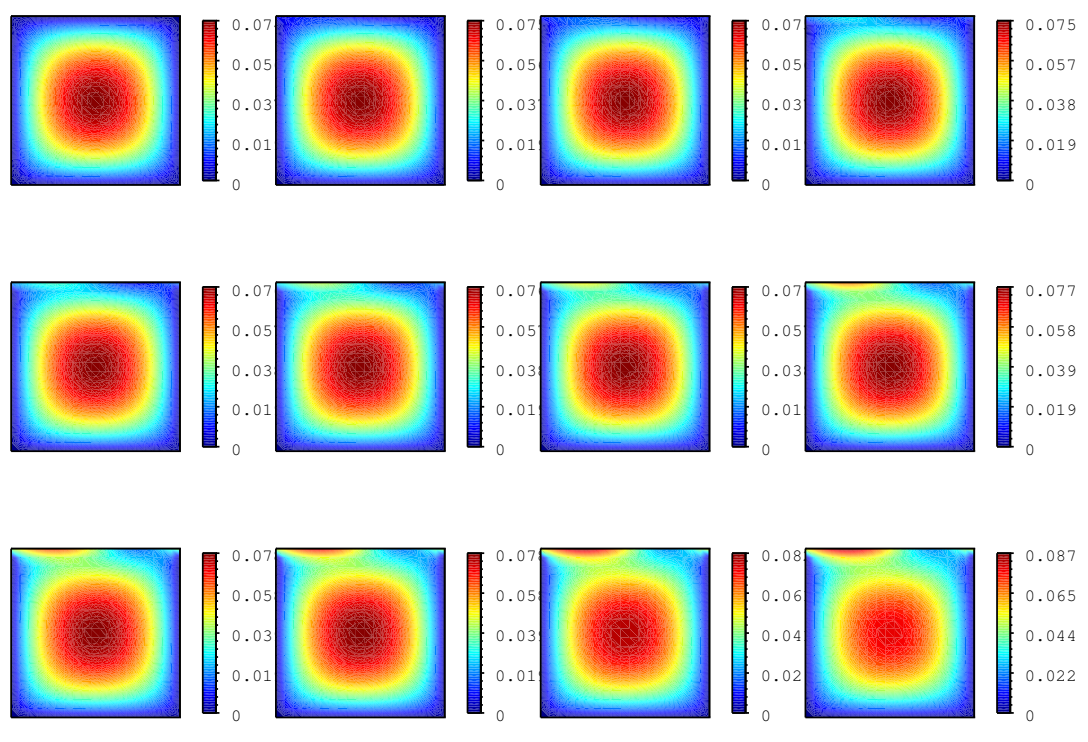

Figure 13: A few snapshots of the direct solution $u^{\theta_{0}}\left(\cdot, t_{n}\right)$ on $\Omega \times[0,5]$ for $\boldsymbol{\theta}_{0}=(0.27,0.92,0.23)^{T}, n=1, \ldots, 12$.

\section{Concluding remarks}

In this paper, we proposed a non-intrusive method for reduced-order modeling of parametrized time-dependent PDEs where the governing equations, the initial and time-dependent boundary conditions are parametrized. The key idea was to represent the reduced-order model as the sum of two terms. The first term was chosen as the approximate solution of an auxiliary parabolic 

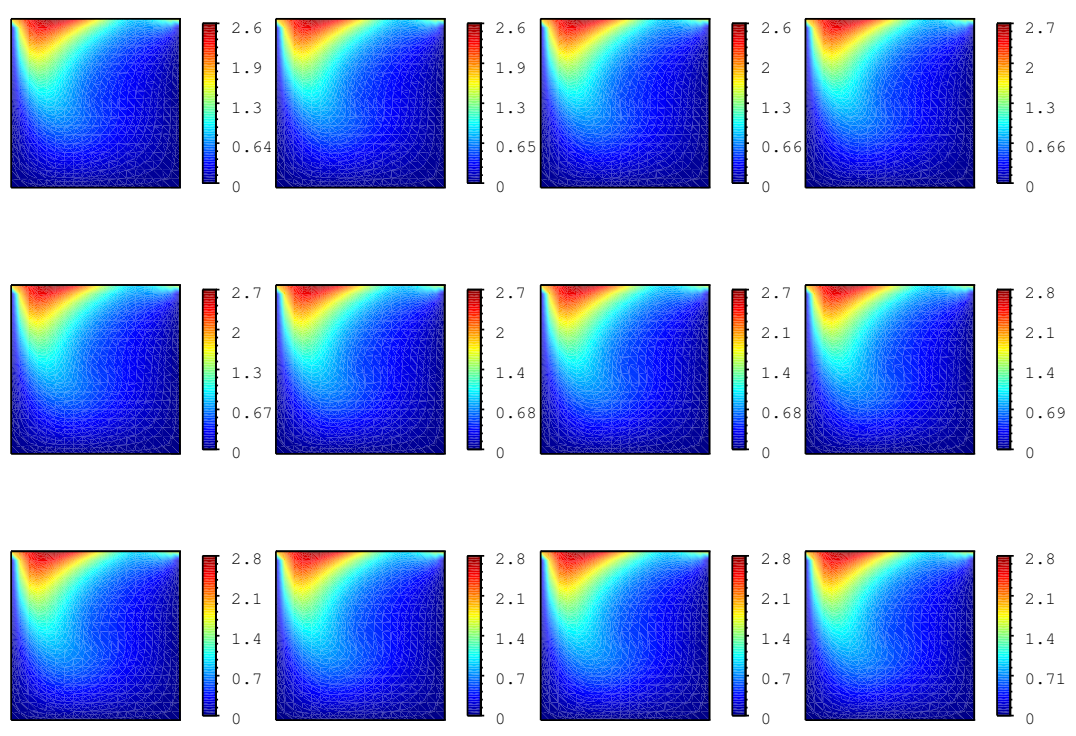

Figure 14: A few snapshots of the direct solution $u^{\theta_{0}}\left(\cdot, t_{n}\right)$ on $\Omega \times[0,5]$ for $\boldsymbol{\theta}_{0}=(0.27,0.92,0.23)^{T}, n=90, \ldots, 101$.
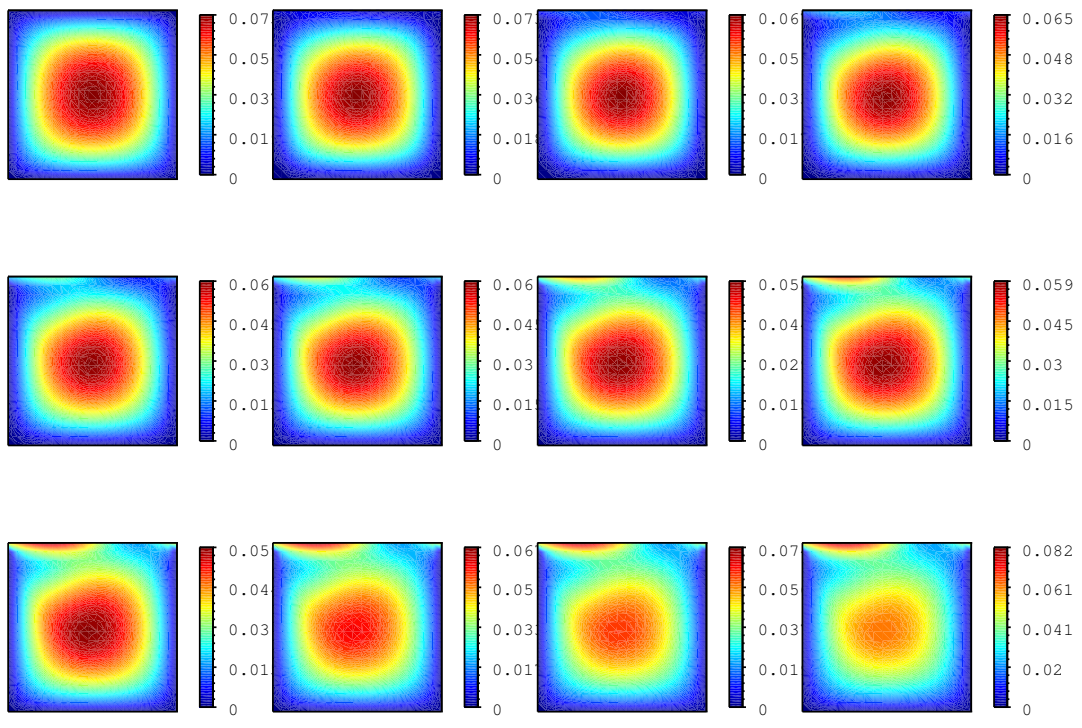

Figure 15: A few snapshots of the ROM solution $\widehat{u}^{\theta_{0}}\left(\cdot, t_{n}\right)$ on $\Omega \times[0,5]$ for $\boldsymbol{\theta}_{0}=(0.27,0.92,0.23)^{T}, n=1, \ldots, 12$. 

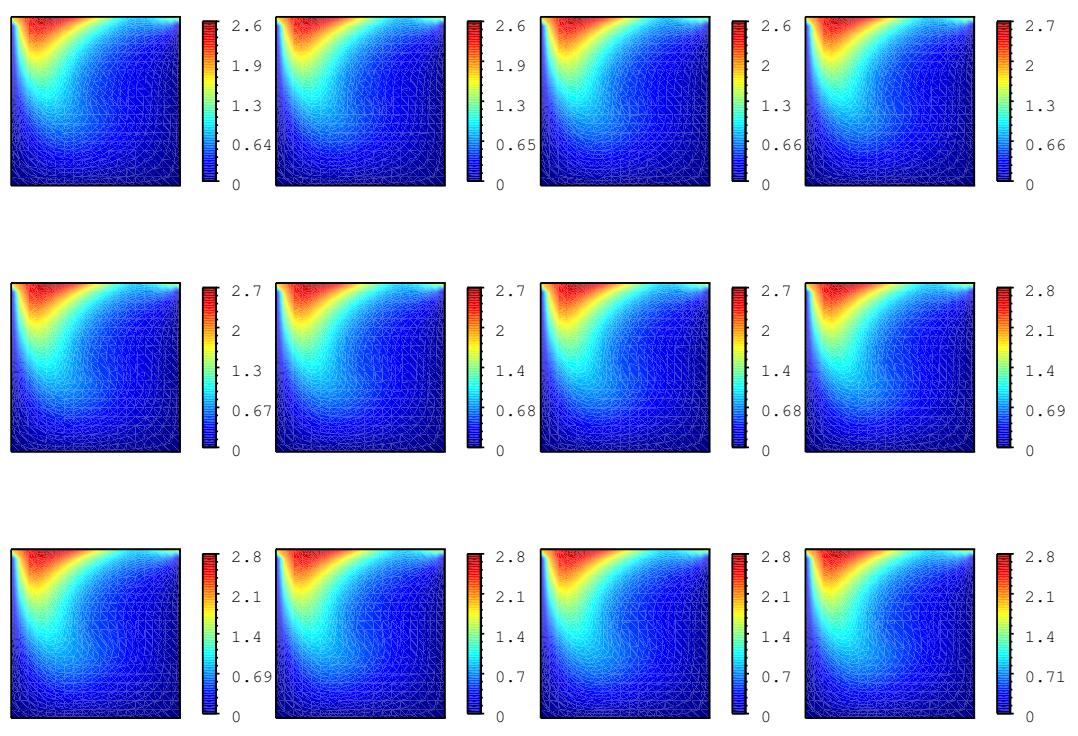

Figure 16: A few snapshots of the ROM solution $\widehat{u}^{\theta_{0}}\left(\cdot, t_{n}\right)$ on $\Omega \times[0,5]$ for $\boldsymbol{\theta}_{0}=(0.27,0.92,0.23)^{T}, n=90, \ldots, 101$

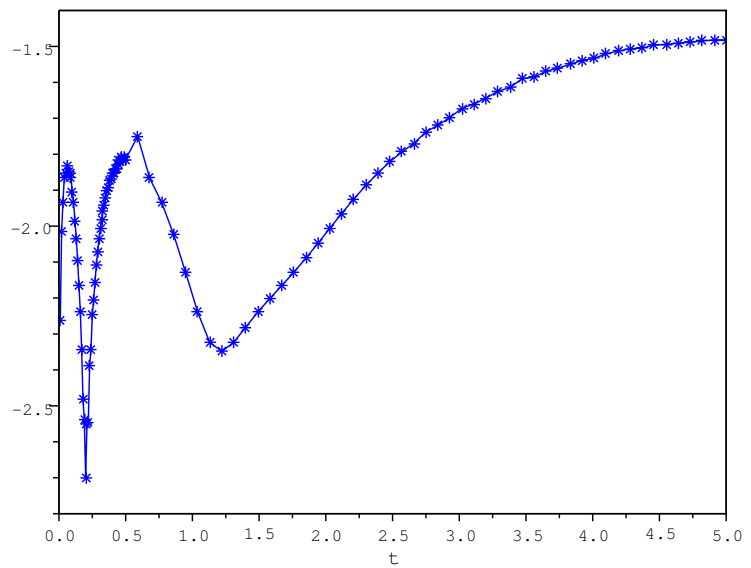

Figure 17: Normalized $L^{2}$ error $\mathcal{E}\left(t_{n}\right)$ in $\log _{10}$ scale between the ROM and direct solutions for $\boldsymbol{\theta}_{0}=(0.27,0.92,0.23)^{T}$, $n=1, \ldots, N$.

linear PDE which enforces satisfaction of the boundary and initial conditions, while the second term is a linear combination of a tensor product of adapted spatial and temporal basis functions 


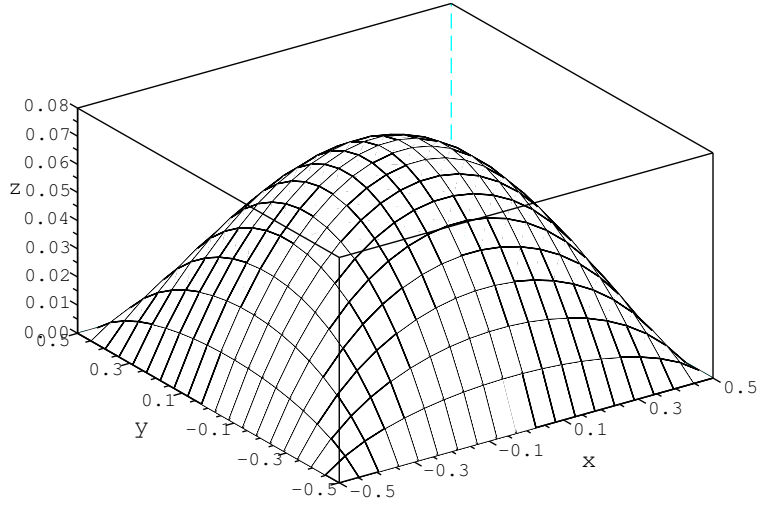

$u^{\theta_{0}}(\cdot, 0)$

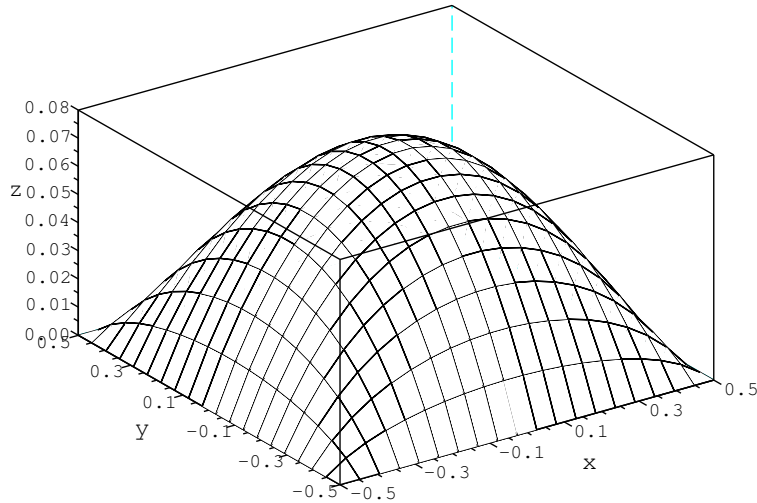

$\widehat{u}^{\theta_{0}}(\cdot, 0)$

Figure 18: 3D representation of the direct solution (left side) and of the ROM one (right side) at time $t=0$.

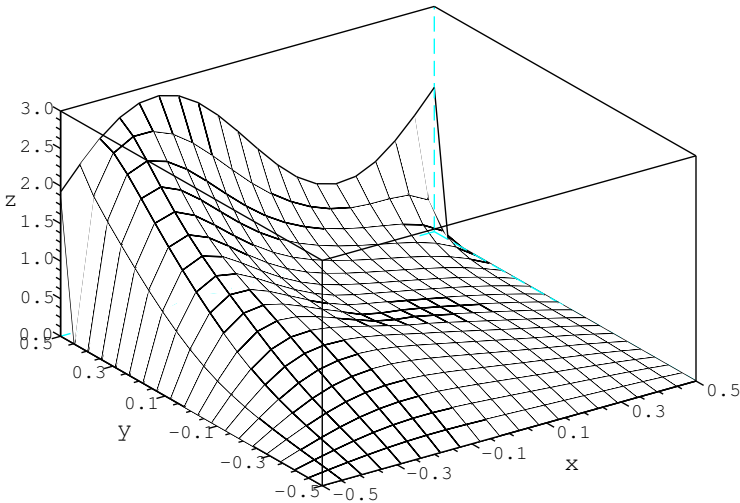

$u^{\theta_{0}}(\cdot, T)$

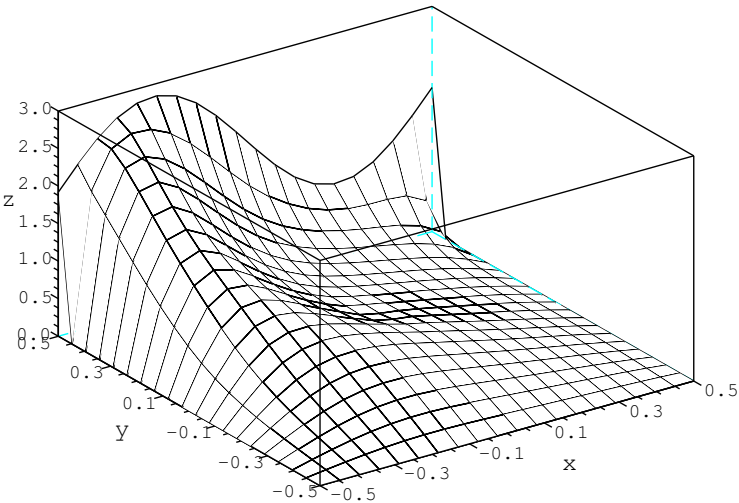

$\widehat{u}^{\theta_{0}}(\cdot, T)$

Figure 19: 3D representation of the direct solution (left side) and of the ROM one (right side) at time $t=T$.

obtained using a two-level POD method. The ability of this approach to accurately reproduce the solutions has been numerically validated for unsteady parametrized Burgers' and convectionreaction-diffusion models. We also provide an error estimate for the reduced-order model used to approximate the solution of the auxiliary parabolic PDE. 


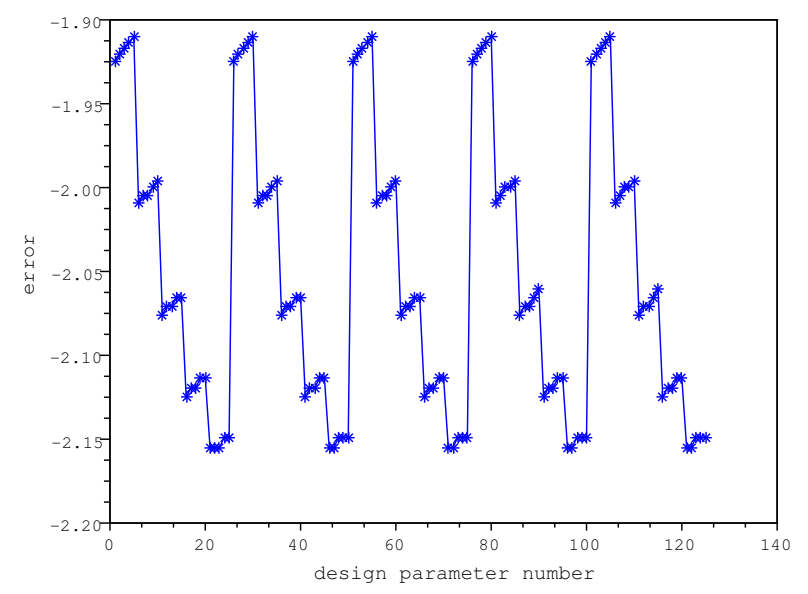

Figure 20: Mean $L^{2}$ errors $E(\boldsymbol{\theta})$ (eq. (84)) in $\log _{10}$ scale, for $\boldsymbol{\theta}$ belonging to the DoCE.

It would be of interest to develop greedy versions of the proposed approach to improve computational efficiency further (see [10] for a detailed exposition of the ROM-greedy algorithm for stationary parametrized problems, and [27, 28] for more general considerations on greedy approaches). It is also of interest to investigate the application of the proposed numerical schemes to solve PDEs that are randomly parametrized (see [29] for ongoing work on this topic). We would like to mention here that the method proposed in this paper can be directly applied to randomly parametrized PDEs since the final reduced-order approximation given by (65) can be efficiently postprocessed to estimate the statistical moments of the solution given the joint probability density function of the parameters. The error estimate provided in this work only applies for the approximation to the auxiliary parabolic PDE and not the original parametrized PDE. Further work is required to establish error estimates for the full non-linear problem. Furthermore, it could be useful to study numerically the influence of the different ROM parameters (number of modes, size of the samplings, etc) on the level of accuracy of the approximate solution. It is also expected that the proposed ROM method may find applications to optimal control theory problems with complex time-dependent boundary conditions.

\section{Acknowledgements}

This research was supported by the United Kingdom Engineering and Physical Sciences Research Council (EPSRC) Grant No. EP/F006802/1. 


\section{Nomenclature}

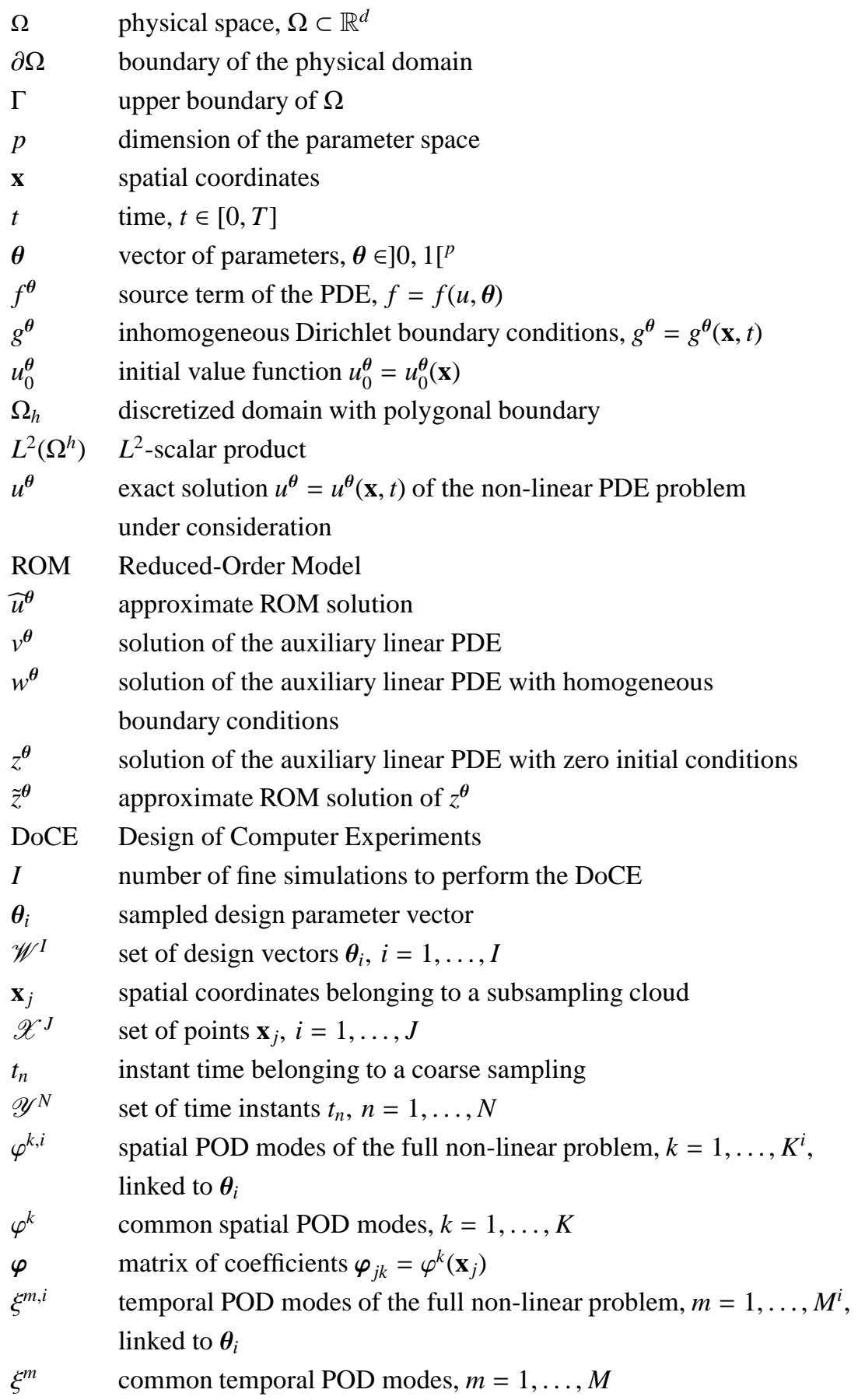




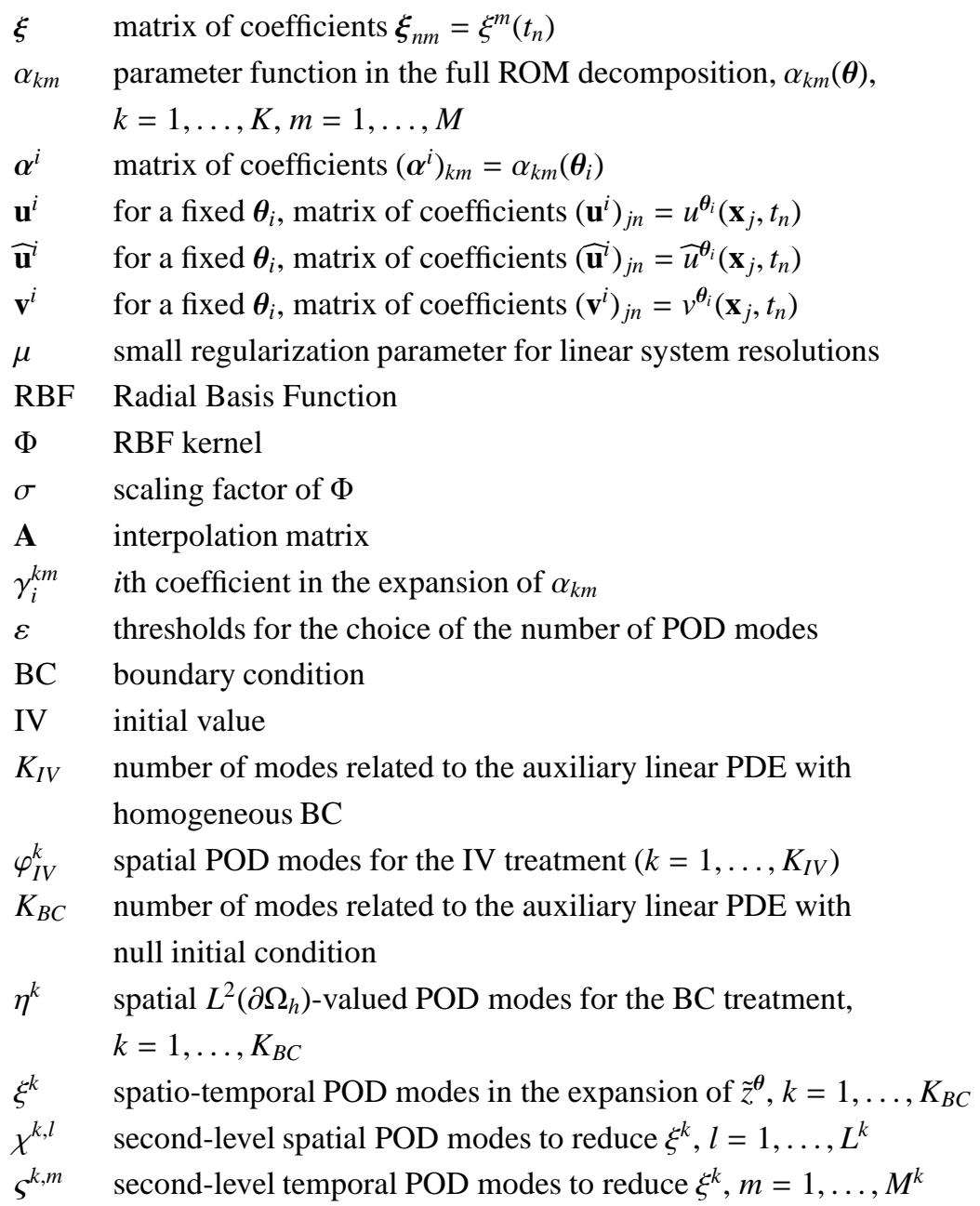

\section{References}

[1] K. Ito and S. S. Ravindran, "A reduced-order method for simulation and control of fluid flows," Journal of Computational Physics, 143: 403-425 (1998).

[2] A. J. Keane and P. B. Nair, Computational Approaches for Aerospace Design, John-Wiley and Sons, 2005.

[3] T. Bui-Thanh, K. Willcox, and O. Ghattas, "Model reduction for large-scale systems with high-dimensional parametric input space," SIAM Journal on Scientific Computing, 30(6):3270-3288 (2008).

[4] M. A Grepl, Y. Maday, N. C. Nguyen, and A. T. Patera, "Efficient reduced-basis treatment of nonaffine and nonlinear partial differential equations," Mathematical Modeling and Numerical Analysis (M2AN), 41(3):575-605 (2007).

[5] P. A. LeGresley, "Application of Proper Orthogonal Decomposition (POD) to Design Decomposition Methods," Ph.D. Dissertation, Stanford University, October 2005.

[6] Y. Maday, A. T. Patera, and G. Turinici, "A priori convergence theory for reduced-basis approximations of singleparameter elliptic partial differential equations," Journal of Scientific Computing, 17:437-446 (2002).

[7] C. Prud'homme, D. Rovas, K. Veroy, Y. Maday, A.T. Patera, and G. Turinici, "Reliable real-time solution of pa- 
rameterized partial differential equations: reduced-basis output bounds," Journal of Fluids Engineering 124:70-80 (2002).

[8] K. Veroy and A. T. Patera, "Certified real-time solution of the parameterized steady incompressible Navier-Stokes equations: Rigorous reduced-basis a posteriori error bounds," International Journal for Numerical Methods in Fluids, 47:773-788 (2005).

[9] S. Deparis and G. Rozza, "Reduced basis method for multi-parameter-dependent steady Navier-Stokes equations: Applications to natural convection in a cavity," Journal of Computational Physics, 228(12):4359-4378 (2009).

[10] C. Audouze, F. De Vuyst and P. B. Nair, "Reduced-order modeling of parameterized PDEs using timespace-parameter Principal Component Analysis," International Journal for Numerical Methods in Engineering, 80(8):1025-1057 (2009).

[11] M. D. Gunzburger, J. S. Peterson and J. N. Shadid, "Reduced-order modeling of time-dependent PDEs with multiple parameters in the boundary data," Computer Methods in Applied Mechanics and Engineering 196:1030-1047 (2007).

[12] A. Hay, J. Borggaard, I. Akhtar and D. Pelletier, "Reduced-order models for parameter dependent geometries based on shape sensitivity analysis," Journal of Computational Physics, 229(4):1327-1352 (2010).

[13] N.-C. Nguyen, G. Rozza, A. T. Patera, "Reduced basis approximation and a posteriori error estimation for the time-dependent viscous Burgers equation”, Calcolo (2009) 46: 157-185.

[14] S. Chaturantabut, D. C. Sorensen, "Discrete Empirical Interpolation for nonlinear model reduction", Technical Report: CAAM, Rice University (2009).

[15] I. M. Sobol, “On the systematic search in a hypercube," SIAM Journal on Numerical Analysis 16(5):790-793, (1979).

[16] T. J. Santner, B. J. Williams and W. I. Notz, The Design and Analysis of Computer Experiments, Springer Verlag, New York, 2003

[17] L. Sirovich, "Turbulence and the dynamics of coherent structures: Parts I-III," Quarterly of Applied Mathematics, 45(3):561-590 (1987).

[18] G. Berkooz, P. Holmes, and J.L. Lumley, "The proper orthogonal decomposition in the analysis of turbulent flows," Annual Review of Fluid Mechanics 25:539-575 (1993).

[19] P. Holmes, J.L. Lumley, and G. Berkooz, Turbulence, Coherent Structures, Dynamical Systems and Symmetry, Cambridge University Press: Cambridge, 1996.

[20] C. Audouze, F. Daïm, F. De Vuyst and P. Laurent-Gengoux, "Space-time proper orthogonal decompositions and radial basis functions for metamodeling of time-dependent fluid flow design analysis," research report ECP RR0703, 2007.

[21] D. J. Lucia and P. S. Beran, "Projection methods for reduced order models of compressible flows, " Journal of Computational Physics 188(1):252-280 (2003).

[22] R. Ghanem, P. Spanos, Stochastic Finite Elements: A Spectral Approach, Springer-Verlag, New York, 1991.

[23] L. C. Evans, Partial Differential Equations, American Mathematical Society, 4th edition, 2008.

[24] W. Rudin, Functional Analysis, Mc Graw Hill, 1973.

[25] R. Bellman, "The stability of solutions of linear differential equations," Duke Math. J., 10:643-647 (1943).

[26] P. Pettersson, G. Iaccarino, J. Nordström, "Numerical analysis of the Burgers equation in the presence of uncertainty", Journal of Computational Physics 228 (2009), 8394-8412.

[27] T. Bui-Thanh, K. Willcox, and O. Ghattas, "Model reduction for large-scale systems with high-dimensional parametric input space," SIAM Journal on Scientific Computing, 30(6):3270-3288 (2008).

[28] P. B. Nair, A. Choudhury, and A. J. Keane, "Some greedy learning algorithms for sparse regression and classification with Mercer kernels," Journal of Machine Learning Research 3:781-801 (2002).

[29] C. Audouze, P. Hakansson, F. De Vuyst, P. B. Nair, "Reduced-order modeling of randomly parameterized PDEs," 4th European Conference on Computational Mechanics, Paris, France, May 16-21, 2010. 